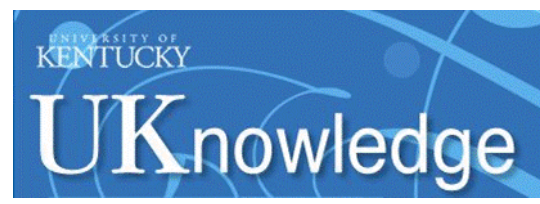

University of Kentucky

UKnowledge

3-1-1999

\title{
Megamaser Disks in Active Galactic Nuclei
}

\author{
John F. Kartje \\ University of Chicago \\ Arieh Königl \\ University of Chicago \\ Moshe Elitzur \\ University of Kentucky, moshe@pa.uky.edu
}

Follow this and additional works at: https://uknowledge.uky.edu/physastron_facpub

Part of the Astrophysics and Astronomy Commons, and the Physics Commons

Right click to open a feedback form in a new tab to let us know how this document benefits you.

\section{Repository Citation}

Kartje, John F.; Königl, Arieh; and Elitzur, Moshe, "Megamaser Disks in Active Galactic Nuclei" (1999).

Physics and Astronomy Faculty Publications. 218.

https://uknowledge.uky.edu/physastron_facpub/218

This Article is brought to you for free and open access by the Physics and Astronomy at UKnowledge. It has been accepted for inclusion in Physics and Astronomy Faculty Publications by an authorized administrator of UKnowledge. For more information, please contact UKnowledge@lsv.uky.edu. 
Megamaser Disks in Active Galactic Nuclei

Digital Object Identifier (DOI)

http://dx.doi.org/10.1086/306824

\section{Notes/Citation Information}

Published in The Astrophysical Journal, v. 513, no. 1, p. 180-196.

(C) 1999. The American Astronomical Society. All rights reserved.

The copyright holder has granted permission for posting the article here. 


\title{
MEGAMASER DISKS IN ACTIVE GALACTIC NUCLEI
}

\author{
JOHN F. KARTJE AND ARIEH KÖNIGL \\ Department of Astronomy and Astrophysics and Enrico Fermi Institute, University of Chicago, 5640 South Ellis Avenue, Chicago, IL 60637; \\ kartje@jets.uchicago.edu, arieh@jets.uchicago.edu \\ AND \\ Moshe Elitzur \\ Department of Physics and Astronomy, University of Kentucky, Lexington, KY 40506; moshe@pa.uky.edu \\ Received 1997 October 8; accepted 1998 October 13
}

\begin{abstract}
Recent spectroscopic and VLBI-imaging observations of bright extragalactic $\mathrm{H}_{2} \mathrm{O}$ maser sources have revealed that the megamaser emission often originates in thin circumnuclear disks near the centers of active galactic nuclei (AGNs). Using general radiative and kinematic considerations and taking account of the observed flux variability, we argue that the maser emission regions are clumpy, a conclusion that is independent of the detailed mechanism (X-ray heating, shocks, etc.) driving the collisionally pumped masers. We examine scenarios in which the clumps represent discrete gas condensations (i.e., clouds) and do not merely correspond to velocity irregularities in the disk. We show that even two clouds that overlap within the velocity-coherence length along the line of sight could account (through selfamplification) for the entire maser flux of a high-velocity "satellite" feature in sources like NGC 4258 and NGC 1068, and we suggest that cloud self-amplification likely contributes also to the flux of the background-amplifying "systemic" features in these objects. Analogous interpretations have previously been proposed for water maser sources in Galactic star-forming regions. We argue that this picture provides a natural explanation of the time-variability characteristics of extragalactic megamaser sources and of their apparent association with Seyfert 2-like galaxies. We also show that the requisite cloud space densities and internal densities are consistent with the typical values of nuclear (broad emission line region type) clouds.

We examine two scenarios of clumpy disks in which the maser emission is excited by a central continuum source. This excitation mechanism was first considered in the context of megamaser disks by Neufeld \& Maloney, but our proposed models are clearly distinct from their warped, homogeneous disk interpretation. In our first scenario we consider an annular disk (or "ring") whose inner edge corresponds to the innermost radius of the observed maser distribution and whose mass is dominated by the clumped, high-density gas component. The shielding of the high-energy continuum, which is required in order that the gas remain molecular, can be provided in this case by the dusty clouds themselves. We show that even the simplest version of this model, in which the disk is flat and the continuum radiation reaches the masing clouds through the plane of the disk, can account for the maser observations in NGC 1068. We point out the striking similarities between the maser ring properties as interpreted with this model and the inferred characteristics of the circumnuclear disk in the Galactic center, and we briefly discuss the implications of such rings for the AGN accretion disk paradigm.

Our second scenario is motivated by the apparent warps observed in some of the imaged megamaser disks and by our finding that the flat-disk version of the irradiated ring scenario could apply to a source like NGC 4258 only if the water abundance in the masing clouds were higher than the value implied by equilibrium photoionization-driven chemistry. This scenario is based on the disk-driven hydromagnetic wind model originally proposed to account for the molecular "tori" in Seyfert 2-like galaxies and for several other observed phenomena in AGNs. In this picture, the wind uplifts (by its ram pressure) and confines (by its magnetic pressure) dense clouds fragmented from the disk, which mase after they become exposed to the central radiation field. Much of the requisite continuum shielding can be provided in this case by the dusty portions of the wind. We show that comparatively massive clouds that move in lowaltitude, nearly circular orbits could be shielded in this way, and we suggest that an apparent warp in the maser distribution might arise under these circumstances from an observational selection effect induced by the strong vertical density stratification that characterizes a centrifugally driven wind. We construct a self-contained illustrative model where the wind transports the bulk of the disk angular momentum, and we show that it is consistent with the data for NGC 4258 as well as with the advectiondominated accretion flow interpretation of the spectrum in this source.

Subject headings: galaxies: individual (NGC 1068, NGC 4258) - galaxies: nuclei - galaxies: Seyfert masers
\end{abstract}

\section{INTRODUCTION}

Strong $22 \mathrm{GHz}$ water maser emission has now been detected toward the nuclei of some 20 active galaxies. These "megamaser" sources are generally associated with Seyfert
2 and LINER galaxies whose nuclei are hidden by a fairly large column of optically obscuring and X-ray-absorbing gas (Braatz, Wilson, \& Henkel 1996, 1997). There is mounting evidence, from spectral measurements and VLBI 
imaging, that in many (if not all) of these objects at least part of the emission originates in a rotating circumnuclear disk on scales of $\sim 0.1-1 \mathrm{pc}$. The best examples to date are provided by NGC 4258 (e.g., Miyoshi et al. 1995; Greenhill et al. 1995b) and NGC 1068 (e.g., Greenhill et al. 1996; Greenhill \& Gwinn 1997), where the imaged maser spots are arranged in a thin and apparently slightly warped disk. In both sources one detects features that are located on the near side of the disk at the vicinity of its apparent inner edge and have line-of-sight velocities that are close to the systemic velocity of the galaxy, as well as high-velocity "satellite" features that are strung out along the projected major axis (the midline) of the disk on either side of the nucleus (where they give rise to red- and blueshifted emission, respectively). In NGC 4258 the systemic maser components lie near $r_{\text {in }} \approx 0.13 \mathrm{pc}$ (assuming a distance $D=6.4$ $\mathrm{Mpc}$ ) and the high-velocity features lie between $\sim 0.16$ and $\sim 0.26 \mathrm{pc}$; in NGC 1068 the inner edge is at $r_{\mathrm{in}} \approx 0.65 \mathrm{pc}$ (for $D=15 \mathrm{Mpc}$ ) and the radii of the high-velocity components span the range $\sim 0.65-1.1 \mathrm{pc}$. In the case of NGC 4258 the emission is dominated by the low-velocity features, which are clustered near the line of sight to the center (the center line); in NGC 1068, the redshifted high-velocity features dominate the measured flux and the emission from the inner edge is not confined to the vicinity of the center line (in fact, a full quarter of the inner edge of the disk appears to be masing in this source, exhibiting a linear increase of the line-of-sight velocity $v_{\text {los }}$ with projected distance from the center). The variation of the line-of-sight velocity of the high-velocity features in NGC 4258 with projected distance from the center indicates a Keplerian disk around a central mass of $\sim 3.5 \times 10^{7} M_{\odot}$; the rotating disk interpretation in this source is further supported by measurements of the apparent centripetal acceleration of the systemic masers (Haschick, Baan, \& Peng 1994; Greenhill et al. 1995a). In NGC 1068 the $v_{\text {los }}$ curve of the high-velocity features exhibits a sub-Keplerian drop with distance, and the inferred central mass is $\gtrsim 10^{7} M_{\odot}$.

Similar results have been obtained in several other sources, although the data have often been more sketchy. In the case of NGC 4945 (Greenhill, Moran, \& Herrnstein 1997b), red- and blueshifted maser features, symmetrically positioned with respect to the systemic feature (at projected distances $\gtrsim 0.2 \mathrm{pc}$ ) have been imaged and a central mass $\gtrsim 10^{6} M_{\odot}$ has been inferred. A possible maser disk has also been found in NGC 3079 (Trotter et al. 1998); in this case only high-velocity (blue- and redshifted) features, distributed over $\sim 1.7 \mathrm{pc}$, have been identified and their motions are consistent with rotation around a binding mass of $\sim 10^{6}$ $M_{\odot}$. Other likely candidates for an association of $\mathrm{H}_{2} \mathrm{O}$ megamaser emission with a rotating disk include NGC 2639 (Wilson, Braatz, \& Henkel 1995), the Circinus galaxy (Greenhill et al. 1997a), and NGC 5793 (Hagiwara et al. 1997). A noteworthy aspect of some of these apparent disks is that they are misaligned with the local symmetry axis. This is particularly pronounced in the case of NGC 1068, where the projected maser disk axis is tilted at an angle of $\sim 35^{\circ}$ with respect to the inner radio jet, which in turn is nearly perpendicular to the plane of the circumnuclear gas distribution (see Gallimore, Baum, \& O'Dea 1996a, 1997; Tacconi et al. 1997). A similar situation is indicated in NGC 3079 , where the apparent maser disk is tilted by $\sim 45^{\circ}$ with respect to the nuclear radio jet.

The imaged $\mathrm{H}_{2} \mathrm{O}$ maser systems are a unique diagnostic of the mass distribution in the centers of active galactic nuclei (AGNs). In particular, the high mass concentrations inferred in sources like NGC 4258 currently provide perhaps the strongest argument for the presence of massive black holes in galactic nuclei (e.g., Maoz 1995, 1998; Rees 1998). By combining centripetal-acceleration and propermotion measurements of the systemic maser spots, one can, in principle, also derive distance ladder-independent values for the distances of these sources (Herrnstein et al. 1997b). But the maser observations also constitute a powerful tool for probing the nature of circumnuclear disks in AGNs. In fact, since the bolometric luminosity in these sources most likely derives from accretion, and since nuclear jets have been detected in many of these objects, the inferred physical conditions in the maser emission regions may have direct implications for the study of accretion and outflow processes in AGNs.

There have been two distinct interpretations of the origin of AGN megamaser disks, based on two alternative excitation mechanisms. Although there is general agreement that the masers are pumped collisionally, in one picture the maser emission arises from the irradiation of the disk by the central continuum source (e.g., Neufeld, Maloney, \& Conger 1994), whereas in the alternative interpretation the masers originate in shock waves that propagate through the disk (e.g., Maoz \& McKee 1998, hereafter MM98). In the disk-irradiation scenario it has been suggested that the disk becomes exposed to the central radiation field because of warping, so that the innermost radius $r_{\text {in }}$ from which maser emission is observed effectively coincides with the onset radius of the warp (Neufeld \& Maloney 1995). The location of the latter is, in principle, determined by the physical mechanism that triggers the warping (e.g., radiation pressure-induced warping instability-Maloney, Begelman, \& Pringle 1996a - or an orbiting massive objectPapaloizou, Terquem, \& Lin 1998). In the shock scenario, the high-velocity emission has been attributed to spiral shocks arising from the steepening of self-sustaining waves that, in the case of a non-self-gravitating, nearly Keplerian disk, are triggered by external forcing (MM98). The trailing spiral shocks envisioned in this model provide a natural explanation of both the quasi-regular spacing of the highvelocity features in sources like NGC 4258 and NGC 1068 and the tendency of the redshifted features to dominate the blueshifted maser emission in most of the objects observed to date.

In the models considered so far in the literature the disk was taken to be essentially homogeneous, and this assumption was used in estimating the mass accretion rate through the maser emission region. In the case of NGC 4258, Neufeld \& Maloney (1995) considered an irradiated, viscous accretion disk and concluded that a rest mass conversion efficiency of $\sim 0.25 / \alpha$ (where $\alpha \leq 1$ is the Shakura \& Sunyaev 1973 viscosity parameter) was needed to account for the bolometric luminosity $L_{\mathrm{bol}} \approx 10^{42} \mathrm{ergs} \mathrm{s}^{-1}$ of the central source (Wilkes et al. 1995; Chary \& Becklin 1997). This efficiency is a factor $\sim 250$ higher than the value inferred in this object from an advection-dominated accretion flow (ADAF) interpretation of its observed spectrum (Lasota et al. 1996; Chary \& Becklin 1997; Herrnstein et al. 1998a). Although the latter estimate applies to disk regions that are significantly smaller than the maser emission zone, the two values are mutually inconsistent if the disk has been accreting in a steady manner over the last few million years 
(Neufeld \& Maloney 1995). The ADAF-implied value also appears to be incompatible with the radiation-induced warping instability model for this galaxy (Maloney et al. 1996a). The spiral shock model, on the other hand, is evidently consistent with the steady state accretion rate $\left(\sim 2 \times 10^{-2} \alpha M_{\odot} \mathrm{yr}^{-1}\right.$; see Herrnstein et al. 1998a) implied by the ADAF interpretation (MM98).

In this paper we take a closer look at the constraints imposed by the observed maser emission on AGN disk models. In $\S 2$ we argue, based on general radiative and kinematic considerations, as well as on the observed flux variability characteristics, that the maser emission regions are clumpy. The likelihood of clumping, which is also indicated by the spectroscopic and imaging data, has already been recognized before (e.g., MM98) and could be associated with either velocity irregularities or density inhomogeneities. The implications of a turbulent velocity field to the observed maser spectra have been considered by Wallin, Watson, \& Wyld $(1998,1999)$. Here we focus on the possibility that distinct gas condensations (clouds) are a major (or even dominant) component of the disk and discuss the expected properties of the resulting maser emission. The motivation for this study is strengthened by our finding that the cloud parameters implied by the maser observations are consistent with those deduced by independent means but on roughly similar scales for optical/UV-emitting clouds in Seyfert galaxies (the so-called broad emission line region [BELR] clouds).

In $\S 3$ we discuss the conditions for efficient water maser emission, starting with the general case of a collisionally pumped gas and then specializing to irradiated clouds in an AGN environment. Although the masing clumps could, in principle, be excited in shocks as in the MM98 model, we concentrate in this paper on scenarios that are based on the alternative possibility of radiative excitation. We construct two such models of irradiated clumpy disks that are qualitatively different from the homogeneous disk model of Neufeld \& Maloney (1995). In the first such model (§ 4) we consider a "minimalist" scenario in which the central continuum radiation reaches the clouds through the plane of the disk: in this case there is, in principle, no need to appeal to warping as in the homogeneous irradiated disk model. In this picture the megamaser disk has a physical inner edge at $r_{\text {in }}$ and is therefore better described as a "ring" (or "annulus"). ${ }^{1}$ Furthermore, because of the small cloud volume filling factor, the estimated disk mass is much lower than that deduced from a homogeneous disk model. Focusing on the case of NGC 1068, we discuss the striking similarity between the maser ring as interpreted in this picture and the circumnuclear dust ring (the so-called circumnuclear disk, or CND) observed in the center of our own galaxy (e.g., Genzel, Hollenbach, \& Townes 1994).

In applying the minimalist scenario considered in $\S 4$ to the megamaser disk in NGC 4258, we find that a strictly flat-disk model is untenable unless the water abundance is significantly higher than that implied by local photoionization-driven chemistry. Since the disk in this source appears to be warped (e.g., Herrnstein, Greenhill, \& Moran 1996a), it is natural to consider a scenario in which the maser distribution is not coplanar. As an alternative to the warped disk model of Neufeld \& Maloney (1995) that

\footnotetext{
${ }^{1}$ A similar disk geometry has been adopted also in the Maoz \& McKee (1998) interpretation of NGC 4258.
}

specifically applies to a clumped mass distribution, we present in $\S 5$ an interpretation of the high-velocity features in terms of clouds that are uplifted from the surface of a magnetized accretion disk by a centrifugally driven wind. Such winds have previously been invoked to explain the origin of the molecular "tori" in Seyfert 2-like galaxies and other AGNs (Königl \& Kartje 1994, hereafter KK94), and their interaction with embedded clouds may have additional important ramifications for active galaxies (Kartje \& Königl 1997, 1999). Using a self-contained set of assumptions to represent a "generic" model of this type, we show that this scenario is consistent with the maser observations in a source like NGC 4258 . We also demonstrate that the previously noted discrepancies between the irradiated accretion disk model for NGC 4258 and the ADAF interpretation of its spectrum can, in principle, be resolved if a centrifugally driven wind carries away a significant fraction of the disk angular momentum in the maser emission region. In $\S 6$ we summarize our results and discuss their implications for the origin of maser emission and the nature of accretion disks in AGNs.

\section{MASER FLUXES FROM AGN DISKS}

\subsection{Flux Constraints}

Even with high-resolution VLBI imaging, the beam size is much larger than the typical maser emission region in an AGN. The flux measured from any small source is given by $F=I \Omega$, where $I$ is the intensity and $\Omega$ is the solid angle subtended by the source at the observing position. If $A$ is the observed area of the source and $D$ its distance, then

$$
F=\frac{2 k T_{b}}{\lambda^{2}} \frac{A}{D^{2}}
$$

where $T_{b}$ is the source brightness temperature and $\lambda$ is the wavelength $(\approx 1.35 \mathrm{~cm}$ for the water maser transition). We now specialize to maser features (of arbitrary shape) that lie in a disk whose plane is aligned with the observer. We denote the half-length of the maser emission region along the line of sight by $\ell$ and define the effective aspect ratio $a$ by

$$
a=\frac{\ell}{\sqrt{A / \pi}} .
$$

For $a$ larger than a few the maser is saturated and its brightness temperature can be expressed as $T_{b} \approx 10^{12} a_{10}^{3} \mathrm{~K}$, where $a_{10}=a / 10$ (e.g., Elitzur, McKee, \& Hollenbach 1991, hereafter EMH91). The observed maser flux is then

$$
F=4.7 \times 10^{17} a_{10}\left(\frac{\ell}{D}\right)^{2} \mathrm{Jy} .
$$

For a maser feature at a distance $r$ from the galactic center, corresponding to an angular distance (in milliarcseconds) $\Theta_{\text {mas }}=2.1 \times 10^{8}(r / D)$, this can be rewritten in the form

$$
F=11 \Theta_{\text {mas }}^{2} a_{10}\left(\frac{\ell}{r}\right)^{2} \mathrm{Jy} .
$$

Equations (3) and (4) are quite general, the only assumption being that the pumping conditions are optimal. The pumping, however, may be driven by any mechanism (shocks, X-ray heating, etc.), and the geometry in the plane can be arbitrary. Equation (4) has the added convenience 
that, in VLBI-imaged sources, $\Theta_{\text {mas }}$ is a measurable quantity.

One can derive an upper bound on the ratio $\ell / r$ in equation (4) by using the velocity-coherence requirement for the maser emission region. We assume Keplerian rotation and focus attention on parcels of gas that give rise to a highvelocity maser feature. If one such parcel is located on the disk midline at a distance $r=0.1 r_{0.1}$ pc from the center and has a line-of-sight velocity $v_{\text {los }}$, then another parcel that lies at a radius $r+\delta r$ and is displaced from the midline a distance $y$ will satisfy the coherence requirement $\Delta v_{\text {los }} \lesssim \Delta v / 2$ (where $\Delta v$ is the width of the maser line) so long as

$$
\left|\left(1+\frac{\delta r}{r}\right)^{-1 / 2}\left[1+\frac{y^{2}}{(r+\delta r)^{2}}\right]^{-3 / 4}-1\right| \lesssim \frac{\Delta v}{2 v_{\text {los }}} .
$$

This result implies that the emission region for any given maser feature is confined to a velocity-coherence "box" of half-width (along the midline)

$$
w_{\mathrm{coh}} \approx\left(\frac{\Delta v}{v_{\mathrm{los}}}\right) r \approx 3.1 \times 10^{14} r_{0.1}\left(\frac{\Delta v}{10^{-3} v_{\mathrm{los}}}\right) \mathrm{cm}
$$

and half-length (along the line of sight)

$$
\ell_{\mathrm{coh}} \approx\left(\frac{2 \Delta v}{3 v_{\mathrm{los}}}\right)^{1 / 2} r \approx 8.0 \times 10^{15} r_{0.1}\left(\frac{\Delta v}{10^{-3} v_{\mathrm{los}}}\right)^{1 / 2} \mathrm{~cm}
$$

(cf. MM98). ${ }^{2}$ Equations (5)-(7) again have a rather general validity and, in fact, apply to any masing gas that is distributed in a Keplerian disk.

Equations (4) and (7) can be combined to give

$$
F \lesssim 0.7 a_{10} \Theta_{10}^{2}\left(\frac{\Delta v}{10^{-3} v_{\text {los }}}\right) \mathrm{Jy},
$$

where $\Theta_{10}=\Theta_{\text {mas }} / 10$. The maximum flux from a maser feature would be produced if the emission came from the entire velocity-coherence box. Adopting a slablike geometry for this box, the observed maser emission area is $A \approx 2 w_{\mathrm{coh}}^{2}$ (Elitzur, Hollenbach, \& McKee 1992, hereafter EHM92), and hence $a_{10} \approx 0.125 \ell_{\text {coh }} / w_{\text {coh }}$. Substituting from equations (6) and (7), one gets $a_{10} \approx 3.2\left(\Delta v / 10^{-3} v_{\text {los }}\right)^{-1 / 2}$, so equation (8) becomes

$$
F_{\max } \approx 2.4 \Theta_{10}^{2}\left(\frac{\Delta v}{10^{-3} v_{\text {los }}}\right)^{1 / 2} \mathrm{Jy} .
$$

Specializing to NGC 1068, for which we adopt as fiducial mean parameters $\Theta_{\text {mas }} \approx 10.9$ and $\left(\Delta v / v_{\text {los }}\right) \approx 3.5 \times 10^{-3}$, we infer from equation (9) that $F_{\max } \approx 5.3 \mathrm{Jy}$. This is about an order of magnitude larger than the maximum flux $(\sim 0.4$ Jy) reported for the high-velocity features in this object (e.g., Greenhill et al. 1996). The implication of this result is that only a fraction of the potentially observable volume in this source actually contributes to the maser emission. In the case of NGC 4258 the measured maximum flux in the highvelocity features (e.g., Moran et al. 1995) sometimes approaches the estimated value of $F_{\max }[\sim 1.1 \mathrm{Jy}$, using $\Theta_{\text {mas }} \approx 6.7$ and $\left.\left(\Delta v / v_{\text {los }}\right) \approx 10^{-3}\right]$; however, in this case, too, most of the high-velocity features typically have lower fluxes. There is, in fact, an independent piece of evidence that indicates that the high-velocity maser emission zone in

\footnotetext{
${ }^{2}$ The height of the velocity-coherence box is determined by such factors as the vertical acceleration of the emitting gas, the thickness of the layer in which optimal masing conditions prevail, and the telescope beamwidth. In the models discussed in this paper, the relevant factor typically turns out to be the beamwidth ( $\lesssim 0.5$ mas).
}

NGC 4258 does not make up the entire velocity-coherent region. From equation (3) it is seen that $F \propto a_{10} \ell^{2}$. Taking $a_{10} \propto \ell_{\text {coh }} / w_{\text {coh }}$, using equations (6) and (7), and noting that, for a Keplerian velocity field, $r \propto v_{\text {los }}^{-2}$, one obtains $F \propto$ $v_{\text {los }}^{-4.5}$. If, instead, one assumes that $a_{10}$ is more or less uniform throughout the emission region (as would be the case, for example, for a collection of similar X-rayirradiated clouds), then an even steeper dependence, $F \propto$ $v_{\text {los }}^{-5}$, is obtained. Now, in the first interferometric mapping on 1994 April 26, the emission from the high-velocity features in NGC 4258 did exhibit a trend of $F$ decreasing with $v_{\text {los }}$ (see Fig. 1 of Miyoshi et al. 1995), but the decline was much milder than $v_{\mathrm{los}}^{-4.5}$ (see also Fig. 11 of Nakai et al. 1995). In mappings performed on 1995 January 8 and 1995 May 28 the highest velocity peaks rose sharply, so the flux near the high end of the spectrum was actually increasing with $v_{\text {los }}$ (Herrnstein et al. 1996b), the opposite of the trend predicted by velocity coherence. Hence, independent of the normalization of the pump estimates, one concludes that the line-of-sight maser sizes do not sample the source velocity field and therefore are not directly determined by the disk dimensions.

The inference that the size of the emission region in objects like NGC 1068 and NGC 4258 is independent of that of the velocity-coherence box is consistent with the MM98 model, in which the dimensions of the masing region are determined by the spiral shock characteristics. One can, however, argue, based on measurements of flux variability, that the emission must arise in distinct clumps (representing velocity or density irregularities within the disk) even if the large-scale structure of the masing region is defined by shocks. In the case of NGC 4258, VLBA observations reported by Herrnstein et al. (1996b) have revealed strong (up to a factor of $\sim 3$ ) variations in the flux measured from some of the high-velocity features on timescales of $\Delta t_{\mathrm{var}} \lesssim$ $8 \Delta t_{8}$ months. A similar behavior was also noted in spectroscopic observations of NGC 1068 (Nakai et al. 1995) and NGC 5793 (Hagiwara et al. 1997). Such variability (which is manifested both by increases and decreases in the maser flux) is not expected in a uniform emission region, but it can be readily interpreted in terms of a relative drift (at a transverse speed $\gtrsim R_{c} / \Delta t_{\mathrm{var}} \approx 5 R_{c 13} \Delta t_{8}^{-1} \mathrm{~km} \mathrm{~s}^{-1}$ ) of distinct masing clumps of projected size $R_{c}=10^{13} R_{c 13} \mathrm{~cm}$ that move into (or out of) alignment with each other along the line of sight to the observer (see $\S 2.2$ ). In order for this behavior to be observed, the clump overlap probability must be high (see eq. [17] below), which is equivalent to the cloud covering factor in the emission region being large. This, in turn, explains why the flux from a given feature can occasionally decline without the entire maser emission region fading away: when one pair of clouds drifts out of alignment, another pair is likely to drift into alignment on a similar timescale.

The presence of clumps has also been deduced in sources like NGC 4258 from spectral and imaging observations of the systemic masers (e.g., Haschick et al. 1994; Greenhill et al. 1995a, 1995b; Herrnstein et al. 1997b). It is interesting to note in this connection that the latter features also exhibit strong variability (including a factor of $\sim 10$ flares lasting on the order of 1 month; Greenhill et al. 1997c) that could potentially arise from similar clump alignment effects (see $\S 2.2$ ), although variability in the central radio continuum source may also be relevant in this case (e.g., Herrnstein et al. 1997a). 


\subsection{Flux from Masing Clouds}

We now consider the maser emission from individual clouds that have a Keplerian disklike distribution. We start with the case of saturated masers and then address the unsaturated ones at the end of this subsection. As discussed, for example, by EHM92, strongly amplified masers have a high degree of beaming and therefore have a filamentary appearance. This is true even for spherical masers, whose observed transverse sizes are significantly smaller than their radii on account of amplification-bounded beaming. Cylindrical masers, on the other hand, are matter bounded, so their observed radii are the same as their projected physical sizes. For the sake of simplicity we consider in what follows cylindrical masers of radius $R_{c}$ and half-length $\ell_{c}=a R_{c}$, although it should be borne in mind that the actual physical dimensions of the masing clouds could be characterized by smaller aspect ratios. Real clouds in a differentially rotating disk would probably be elongated at least to some degree in the azimuthal direction and hence may be better represented by cylinders than by spheres.

Focusing on the high-velocity maser features, which we represent as filaments viewed end-on, the flux from an individual cloud is given by

$$
F_{c} \approx 5.0 \times 10^{-6} D_{10}^{-2} R_{c 13}^{2} a_{10}^{3} \mathrm{Jy}
$$

(see eq. [3]), where we set $D_{10}=(D / 10 \mathrm{Mpc})$. This value is much smaller than the flux $(F \gtrsim 0.1 \mathrm{Jy})$ typically measured from the bright high-velocity maser features in AGNs. However, if two cloud emission regions that lie within the velocity-coherence box overlap along the line of sight, the flux from the background cloud can be strongly amplified by the foreground emission region (Deguchi \& Watson 1989; EMH91). The effective brightness temperature $T_{b \text {,eff }}$ for two overlapping masers that are separated by a distance $s$, each characterized by a brightness temperature $T_{b}$ and aspect ratio $a$, is

$$
T_{b, \text { eff }} \approx \frac{16}{11}\left(\frac{s}{a R_{c}}\right)^{2}\left[1+\left(\frac{s}{d_{i}}\right)^{2}\right]^{-1} T_{b},
$$

where

$$
d_{i}=10^{18} a_{10}^{2} \gamma_{5}^{1 / 2} R_{c 13} \mathrm{~cm}
$$

is the interaction distance, expressed in terms of the ratio $\gamma=10^{5} \gamma_{5}$ of the maser saturation intensity to the unsaturated source function (EMH91; see also Elitzur 1992). The corresponding flux is given by

$$
F \approx 7.3 \times 10^{-2} a_{10} D_{10}^{-2} s_{16}^{2}\left(1+10^{-4} s_{16}^{2} d_{i 18}^{-2}\right)^{-1} \mathrm{Jy},
$$

where we put $s_{16}=\left(\mathrm{s} / 10^{16} \mathrm{~cm}\right)$ and $d_{i 18}=\left(d_{i} / 10^{18} \mathrm{~cm}\right)$. Note the interesting result that, in the limit $s \ll d_{i}$, the selfamplified flux is independent of the cloud size (depending on the cloud dimensions only through the aspect ratio a) and is determined primarily by the line-of-sight separation between the clouds $\left(F \propto s^{2}\right)$. It is seen that even two such clouds could, in principle, account for the entire measured flux $F=0.1 F_{0.1}$ Jy of a maser feature if they were separated by a distance greater than

$$
s_{\min } \approx 1.2 \times 10^{16} F_{0.1}^{1 / 2} D_{10} a_{10}^{-1 / 2} \mathrm{~cm}
$$

along the line of sight (expression valid for $s_{\min } \ll d_{i}$ ). The value of $s_{\min }$ would be larger if the cloud emission regions overlapped only partially. On the other hand, the value of $S_{\text {min }}$ could be smaller if more than one interacting pair of masing clouds contributed to the observed flux.
For self-consistency, $s_{\min }$ is required to be $<2 \ell$ coh (eq. [7]). This condition appears to be satisfied in NGC 4258, where (using $D_{10} \approx 0.64, a_{10} \approx 1$, and $F_{0.1} \approx 10$ in eq. [14] and evaluating eq. [7] at $r=0.21 \mathrm{pc}), s_{\mathrm{min}} / \ell_{\mathrm{coh}} \approx 1.4$, as well as in NGC 1068, where (with $D_{10} \approx 1.5, a_{10} \approx 1, F_{0.1} \approx 4$, and $r=0.88 \mathrm{pc}) s_{\min } / \ell_{\text {coh }} \approx 0.27$. The fact that the estimated value of $s_{\text {min }}$ turns out not to be much smaller than the maximum value of $\ell_{\text {coh }}$ suggests that there is a strong observational bias for detecting high-velocity megamaser features in Seyfert 2-like galaxies. In these AGNs the disk rotation axis is nearly perpendicular to the line of sight and the corresponding value of $\ell_{\text {coh }}$ is large: by contrast, AGNs with disks that are viewed at smaller angles to the axis have a comparatively low probability of overlap for masing clouds that satisfy the velocity-coherence constraints. Note that, if cloud "stretching" is tied to the velocity shear in the disk, then a large inclination angle would also be favored by the dependence of the flux on the filament aspect ratio $a$.

One can show that masing clouds with typical parameters that are even moderately elongated are likely to be saturated. The saturation condition for a cylindrical maser can be written as

$$
\frac{\exp \left[a \kappa_{0} R_{c}\right]}{a}=4 \sqrt{\gamma}
$$

where $\kappa_{0}$ is the unsaturated line center absorption coefficient (EMH91). When all parameters except for $a$ are fixed, this relation determines $a_{\text {sat }}$, the minimum aspect ratio for saturation. As an illustration, when the masing gas is characterized by a hydrogen number density of $10^{9} \mathrm{~cm}^{-3}$, temperature of $400 \mathrm{~K}$, maser line width of $1 \mathrm{~km} \mathrm{~s}^{-1}$, and fractional water abundance of $10^{-5}$, then $\kappa_{0} R_{c}=$ $0.8,1.2,2.7$, and 3.7 for $R_{c 13}=0.5,1,5$, and 10, respectively. The solutions of equation (15) are then $a_{\text {sat }} \approx 12.9,8.0,3.0$, and 2.2 , respectively, where we set $\gamma_{5}=1$. The corresponding values for a fractional water abundance of $10^{-4}$ are $\kappa_{0} R_{c}=2.7,3.7,6.1$, and 7.2 and $a_{\text {sat }} \approx 3.0,2.2,1.2$, and 1.0, respectively. (Note that, in order to be considered filamentary, the maser must satisfy $a \gg \max \left\{1, \kappa_{0} R_{c} / 4\right\}$; EMH91.) For comparison, a spherical maser of radius $R_{c}$ saturates when $\kappa_{0} R_{c} \approx 7.5$ (assuming again $\gamma_{5}=1$ ), at which point it appears as a cylinder with an effective aspect ratio of $\sim 2.9$ (see EHM92). These results indicate that masing clouds that are at least somewhat elongated and have $\kappa_{0} R_{c}$-values of the order of a few would be saturated.

In a source like NGC 4258 one can use information on the systemic masers to help assess whether the saturation condition on the high-velocity features is satisfied. Based on the location of the systemic masers relative to the nuclear radio jet and on the apparent correlation between the jet continuum and maser flux densities, it has been inferred that the systemic maser features in this object act as amplifiers of the background radio continuum (e.g., Herrnstein et al. 1997a). On the assumption that each such feature is produced by the same type of filamentary clouds that give rise to the high-velocity emission (except that they are now viewed at a right angle to their axes), the brightness temperature of the systemic masers is found to be $T_{b \text {, sys }} \approx 2.6$ $\times 10^{15}(F / 2 \quad \mathrm{Jy})(a / 2)^{-1}\left(R_{c} / 5 \times 10^{13} \mathrm{~cm}\right)^{-2} \mathrm{~K}$. Setting $T_{b \text {,sys }}=\exp \left(\tau_{\text {sys,tot }}\right) T_{b \text {, cont }}$, where $T_{b \text {, cont }}\left(\lesssim 10^{8} \mathrm{~K}\right.$; Greenhill et al. 1995b; Herrnstein et al. 1998a) is the brightness temperature of the amplified background continuum, we deduce that the total optical depth $\tau_{\text {sys, tot }}$ of the systemic 
masers is $\sim 17.1$. If the maximum systemic maser flux is produced by two fully overlapping clouds then it follows that $\kappa_{0} R_{c} \approx \tau_{\text {sys, tot }} / 4 \approx 4.27$. Now, the total optical depth $\tau_{\mathrm{hi}, \text { tot }}$ for the high-velocity masers is given in terms of the maser brightness temperature $T_{b, \mathrm{hi}} \approx 1.6 \times 10^{15}(\mathrm{~F} / 0.5$ $\mathrm{Jy})\left(R_{c} / 5 \times 10^{13} \mathrm{~cm}\right)^{-2} \mathrm{~K}$ and the excitation temperature $T_{x} \approx 10^{2} \mathrm{~K}$ (e.g., Elitzur 1992) by $\tau_{\mathrm{hi}, \text { tot }} \approx \ln \left(T_{b, \mathrm{hi}} / T_{x}\right)$. Attributing the high-velocity emission to two overlapping filaments viewed end-on, we obtain for the above fiducial values $a \kappa_{0} R_{c} \approx 7.6$, which, when combined with the systemic masers result, implies $a \approx 1.78$. This value is just shy of the saturation aspect ratio $a_{\text {sat }} \approx 1.81$ obtained from equation (15) for $\kappa_{0} R_{c}=4.27$.

From the above estimate we conclude that the saturation assumption for the high-velocity masers in a source like NGC 4258 is probably valid, although it might be only marginally so in clouds with large values of $R_{c}$. (Recall in this connection that an upper bound on $R_{c}$ is provided by the half-width $w_{\text {coh }}$ of the velocity-coherence box, given by eq. [6].) Our interpretation of the high-velocity features in terms of two overlapping filaments would not fundamentally change even if the filaments were unsaturated. In that case the brightness temperature of each individual filament would drop from $\sim 10^{12} a_{10}^{3} \mathrm{~K}$ to $\sim 10^{2} \exp \left(2 a \kappa_{0} R_{c}\right) \mathrm{K}$, but the self-amplification factor would increase from the value given by equation (11) to $\sim \exp \left(2 a \kappa_{0} R_{c}\right)$, resulting in the same final brightness temperature $T_{b, \mathrm{hi}}$. [Note that the two masers would remain unsaturated only if the condition $\left(\pi R_{c}^{2} / 4 \pi s^{2}\right) \exp \left(2 a \kappa_{0} R_{c}\right)>1$ is satisfied, which would be the case only if the cloud separation $s$ is not too large.] Even though the amplification factor in the unsaturated case no longer depends on $s$ (in contrast with eq. [11]), the argument that high-velocity maser features should be seen only at large inclination angles to the disk rotation axis still applies since a comparatively large value of $\ell_{\text {coh }}$ is still required to attain a measurable probability of cloud overlap.

If the systemic maser emission in a source like NGC 4258 arises in two or more overlapping clumps that amplify the background continuum, then their detection probability would also be maximized at large inclination angles. The coherence half-length near the center line of a Keplerian disk viewed along its plane can exceed the midline value (eq. [7]) if the emission originates sufficiently close to the center line. Exactly along the center line all points have the same line-of-sight speed $\left(v_{\text {los }}=0\right)$, whereas for a masing parcel at a radius $r$ where $\Delta v \lesssim v_{\text {los }} \lesssim v_{\mathrm{K}} / 2$ (with $v_{\mathrm{K}}$ denoting the Keplerian rotation speed) the coherence half-length is $\sim\left(\Delta v / 3 v_{\text {los }}\right) r$ (which differs slightly from the expression in MM98); these values may be reduced if the disk plane is tilted with respect to the line of sight. Since the coherence length is maximized along the center line, one would expect the systemic maser emission to peak there; in fact, the maser spectrum in NGC 4258 exhibits a dip at $v_{\text {los }}=0$, which is most naturally attributed to absorption outside the emission region (Watson \& Wallin 1994; MM98; see § 4). The systemic maser emission is thus maximized on either side of the center line, where the velocity-coherence length along the line of sight from the emission point does not reach all the way into the absorption region. For these features the line-of-sight coherence length may still be comparable to, or even exceed, the value associated with the high-velocity masers. Hence, if the high-velocity maser emission originates in overlapping clouds, at least part of the systemic maser emission might too. The motion of individual clouds into or out of alignment along the line of sight (see $\S 2.1$ ) could then also explain some of the strong flux variability exhibited by the systemic masers (e.g., Greenhill et al. 1997c). We address additional aspects of the systemic masers in $\S 4$.

\section{MASING CONDITIONS}

Optimal emission in the $6_{16}-5_{23} 22 \mathrm{GHz}$ maser transition from collisionally pumped water molecules is achieved for hydrogen nuclei densities of $n_{\mathrm{H}} \sim 10^{8}-10^{9}$ $\mathrm{cm}^{-3}$, temperatures of $T \gtrsim 250 \mathrm{~K}$, and fractional water abundances $\quad x\left(\mathrm{H}_{2} \mathrm{O}\right) \equiv n\left(\mathrm{H}_{2} \mathrm{O}\right) / n_{\mathrm{H}} \sim 10^{-5}-10^{-4} \quad$ (e.g., Elitzur 1992). We note in passing that the required densities fall within the inferred characteristic range in BELR clouds (e.g., Baldwin et al. 1995). Neufeld et al. (1994) showed that these conditions are attained in a dusty gas of density $n_{\mathrm{H}} \gtrsim$ $10^{8} \mathrm{~cm}^{-3}$ that is irradiated by a typical AGN X-ray spectrum if the column density $N_{\mathrm{H}}$ exceeds $\sim 10^{23} \mathrm{~cm}^{-2}$ (see also Pier \& Voit 1995; Wallin \& Watson 1997). The thermal structure of a photoionized, optically thin medium is in general determined by the ionization parameter $\Xi \equiv$ $F_{\text {ion }} / p c$, where $F_{\text {ion }}$ is the local ionizing flux and $p=n_{\mathrm{H}} k T$ (with $k$ being Boltzmann's constant) is the gas thermal pressure (modulo a molecular weight factor). In the case of a dusty, molecular gas, which absorbs the low-energy ionizing photons up to an energy that depends on the magnitude of the column density, one can construct an effective ionization parameter that explicitly accounts for the attenuation of the incident ionizing flux $F_{\text {ion,o }}$ and that can be approximated by $\Xi_{\text {eff }} \approx F_{\text {ion, } 0} / N_{\mathrm{H}} p c$ (Maloney, Hollenbach, \& Tielens 1996b). Neufeld et al. (1994) identified the maximum value of $\Xi_{\text {eff }}$ where a stable molecular phase first appears, and Neufeld \& Maloney (1995), in turn, used this value to obtain the minimum pressure that is required (given $F_{\text {ion, } 0}$ and $N_{\mathrm{H}}$ ) for the gas in an irradiated disk to be molecular. In the models considered in this paper it is more convenient to inquire about the minimum shielding column, $N_{\min }$, that must be present for given irradiating flux and gas pressure in order for the gas to be molecular. To derive the gas thermal properties in our particular applications we employ the photoionization code CLOUDY (Ferland 1996) and compute the run of temperature and water abundance as a function of depth into a slab of given (uniform) density that is irradiated by an external continuum flux and is shielded by a dusty gas of column density $N_{\text {shield }}$. The grain and elemental abundances are taken to be those of the local ISM. Figure 1 shows the run of $N_{\min }$ obtained in this way as a function of $p / k$ for clouds (of hydrogen nuclei density $n_{c}$ and temperature $T_{c}$ ) that are located in the $\mathrm{H}_{2} \mathrm{O}$ maser emission regions in NGC 1068 and NGC 4258. For NGC 1068 we adopt the spectrum given by Pier et al. (1994) with $L_{\text {bol }} \approx 4 \times 10^{44} \mathrm{ergs} \mathrm{s}^{-1}$ and set $r=1 \mathrm{pc}$, whereas for NGC 4258 we use the spectral information from Wilkes et al. (1995) and Chary \& Becklin (1997) and set $L_{\mathrm{bol}} \approx 10^{42} \mathrm{ergs}$ $\mathrm{s}^{-1}$ and $r=0.25 \mathrm{pc}$. Approximate polynomial fits to the function $N_{\min }(p)$ for these two cases are given in the caption to Figure 1 . Since $T_{c}$ is determined by the value of the ionization parameter, $N_{\min }$ can be regarded as being just a function of $n_{c}$.

Molecular gas in an AGN can remain dusty beyond the dust sublimation radius $r_{\text {sub }}$, defined as the distance interior to which dust grains exposed to the ambient radiation field are evaporated. The primary significance of dust to AGN 


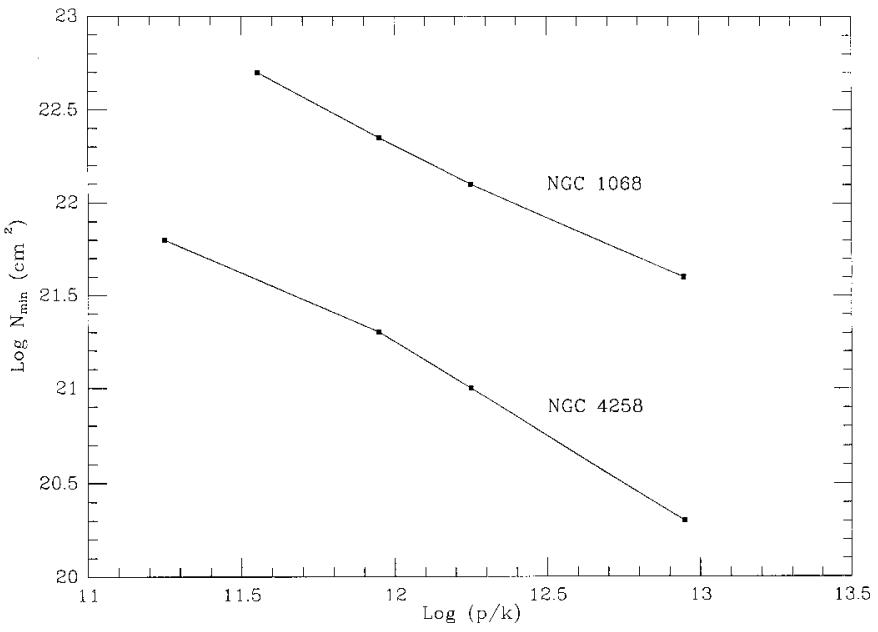

Fig. 1.-Minimum shielding column density of dusty gas that is required for photoionized clouds to be molecular, as a function of the reduced pressure $p / k=n_{\mathrm{H}} T$ of the irradiated clouds. The two curves correspond to clouds that are located at a distance of 1 and $0.25 \mathrm{pc}$ from the central continuum source in NGC $1068\left(L_{\text {bol }} \approx 4 \times 10^{44} \mathrm{ergs} \mathrm{s}^{-1}\right)$ and NGC $4258\left(L_{\text {bol }} \approx 10^{42} \mathrm{ergs} \mathrm{s}^{-1}\right)$, respectively. Fitting these curves by an expression of the form $\log N_{\min }=c_{1}+c_{2} x+c_{3} x^{2}$, where $x=\log (p / k)$, one finds $\left\{c_{1}, c_{2}, c_{3}\right\}=\{47.10,-3.29,0.10\}$ and $\{14.46,1.99,-0.12\}$ for NGC 1068 and NGC 4258, respectively.

maser emission is that, by absorbing UV and soft X-ray photons from the central continuum, it lowers the ionization state and hence the temperature of the gas so that water molecules can survive (or form) at distances from the center where the gas would otherwise be hot and atomic. In the presence of a sufficiently large shielding column the temperature drops below $T \approx 1500 \mathrm{~K}$ and the gas remains (or becomes) molecular (see, e.g., Fig. 1 in Neufeld et al. 1994). At this temperature $\mathrm{H}_{2}$ is efficiently produced by the reaction $\mathrm{H}^{-}+\mathrm{H} \rightarrow \mathrm{H}_{2}+e$, which permits water formation via the reaction network $\mathrm{O}+\mathrm{H}_{2} \rightarrow \mathrm{OH}+\mathrm{H}$; OH $+\mathrm{H}_{2} \rightarrow \mathrm{H}_{2} \mathrm{O}+\mathrm{H}$. Therefore, even if the water abundance in the disk were initially low, it could become comparatively high $\left[x\left(\mathrm{H}_{2} \mathrm{O}\right) \gtrsim 10^{-5}\right]$ after it gets exposed to the (shielded) central continuum. The magnitude of the sublimation radius depends on the detailed spectral energy distribution and scales with the source bolometric luminosity as $L_{\mathrm{bol}}^{1 / 2}$. Its precise value also depends on the grain composition and size distribution (e.g., Laor \& Draine 1993). Using the same prescription as in KK94 (see their Appendix B), we infer $r_{\mathrm{sub}} \approx 0.23\left(L_{\mathrm{bol}} / 4 \times 10^{44} \mathrm{ergs} \mathrm{s}^{-1}\right)^{1 / 2} \mathrm{pc}$ in NGC 1068 and $r_{\mathrm{sub}} \approx 0.015\left(L_{\mathrm{bol}} / 10^{42} \mathrm{ergs} \mathrm{s}^{-1}\right)^{1 / 2} \mathrm{pc}$ in NGC 4258. In a similar manner we deduce $r_{\text {sub }} \approx 0.15\left(L_{\mathrm{bol}} / 10^{44} \mathrm{ergs} \mathrm{s}^{-1}\right)^{1 / 2}$ $\mathrm{pc}$ in NGC 4945 (using the spectral information in Iwasawa et al. 1993) and $r_{\text {sub }} \approx 0.25\left(L_{\text {bol }} / 3 \times 10^{44} \mathrm{ergs} \mathrm{s}^{-1}\right)^{1 / 2} \mathrm{pc}$ in NGC 3079 (using the scaled nuclear bolometric luminosity from Hawarden et al. 1995 together with the inferred spectral shape of NGC 4258). If the maser emission is excited by continuum irradiation then $r_{\text {sub }}$ should not exceed the inner radius $r_{\text {in }}$ of the maser emission region: the observed values of $r_{\text {in }}$ (see $\left.\S 1\right)$ evidently satisfy this inequality for the above sources. $^{3}$

When the column density of dusty gas that shields the continuum source becomes large enough, the cloud tem-

\footnotetext{
${ }^{3}$ The possible relevance of the dust sublimation radius to the location of the inner boundary of the maser emission region was already pointed out by Greenhill et al. (1996) in their discussion of the NGC 1068 VLBI imaging data.
}

perature remains below $250 \mathrm{~K}$ and the water level population inversion required for maser emission does not occur. We propose that, in the context of clumpy disk models, this condition (rather than the transition from molecular to atomic gas invoked in the homogeneous disk model of Neufeld \& Maloney 1995) determines the outermost radius $r_{\text {out }}$ of the maser emission region. Specifically, for a given incident continuum spectrum, the condition $T \approx 250 \mathrm{~K}$ at the upstream edge of a cloud determines the magnitude of the intervening column density, which we denote by $N_{\max }$. For a given gas distribution, this value, in turn, fixes $r_{\text {out }}$. Since the temperature is tied to the value of the effective ionization parameter $\Xi_{\text {eff }}$, which depends on the gas density, $N_{\max }$ is a function of $n_{c}$ (and, like $N_{\min }$, scales roughly as $n_{c}^{-1}$ ).

Clouds located at radii $r<r_{\text {out }}$ have temperatures $T>250 \mathrm{~K}$, so their water molecules are collisionally pumped. Adopting again the filamentary geometry introduced in $\S 2.1$, the maser efficiency in these clouds can be parameterized in terms of the effective emission measure

$$
\xi=0.2 x_{-5}\left(\mathrm{H}_{2} \mathrm{O}\right) n_{c 9}^{2} R_{c 13} / \Delta v_{5}
$$

where $x_{-5}\left(\mathrm{H}_{2} \mathrm{O}\right)=x\left(\mathrm{H}_{2} \mathrm{O}\right) / 10^{-5}, n_{c 9}=\left(n_{c} / 10^{9} \mathrm{~cm}^{-3}\right)$, and $\Delta v_{5}=\left(\Delta v / 10^{5} \mathrm{~cm} \mathrm{~s}^{-1}\right)$ (Elitzur, Hollenbach, \& McKee 1989, hereafter EHM89). Optimal masing conditions obtain for $1 \lesssim \xi \lesssim 100$. For $\xi \gtrsim 100$, the (dusty) cloud column density $\sim 10^{23} \xi \Delta v_{5} /\left(x_{-5} n_{c 9}\right) \mathrm{cm}^{-2}$ becomes too large for $T$ to remain above $\sim 250 \mathrm{~K}^{4}$

\section{MASING RINGS}

In this section we incorporate the masing conditions derived in $\S 3$ into a set of self-consistency constraints for a minimalist model of an irradiated disk, and we demonstrate that this model can account for the $\mathrm{H}_{2} \mathrm{O}$ maser observations in a source like NGC 1068. A schematic illustration of the envisioned scenario is given in Figure 2. We consider a planar ring of inner radius $r_{\text {in }}$ that surrounds the central continuum source. The ring is composed of dusty molecular clumps and possibly also of a dusty interclump medium, but the total column density through the disk is assumed to be sufficiently low that the ionizing radiation can reach the masing clouds through the plane of the ring. For simplicity, we consider a collection of identical cylindrical clouds of density $n_{c}$, radius $R_{c}$, and aspect ratio $a \approx 10$, whose axes are roughly perpendicular to the radius vectors from the center. ${ }^{5}$ We further assume that the clouds are distributed uniformly throughout the disk. Focusing attention on the high-velocity features, which we interpret in terms of overlapping clouds (see $\S 2$ ), we derive an approximate estimate of the minimum number density of clouds $\mathscr{N}_{c, \text { min }}$ that is

\footnotetext{
${ }^{4}$ In the case of a dust-free gas, a similar upper limit on the optical depth is commonly attributed to the fact that, for $\xi \gtrsim 100$, the maser quenches because of infrared photon trapping that tends to thermalize the level populations (EHM89). However, in the case of a dusty gas, Collison \& Watson (1995; see also Wallin \& Watson 1997) have shown that, if the grain and gas temperatures differ by more than $\sim 10 \mathrm{~K}$, the pumping is no longer quenched but instead remains constant with increasing optical depth. Although this condition is generally satisfied for the clouds that we model in this paper, $\xi \approx 100$ is still the relevant upper limit because of the temperature constraint mentioned in the text. In any case, the clouds in the scenarios that we explore are sufficiently small that their individual column densities do not even approach this upper limit.

${ }^{5}$ A more physical characterization of the clouds would have been in terms of their pressure $p$ rather than $n_{c}$ (see Neufeld et al. 1994), but for the narrow range in temperatures that is of interest to us the distinction is not significant.
} 


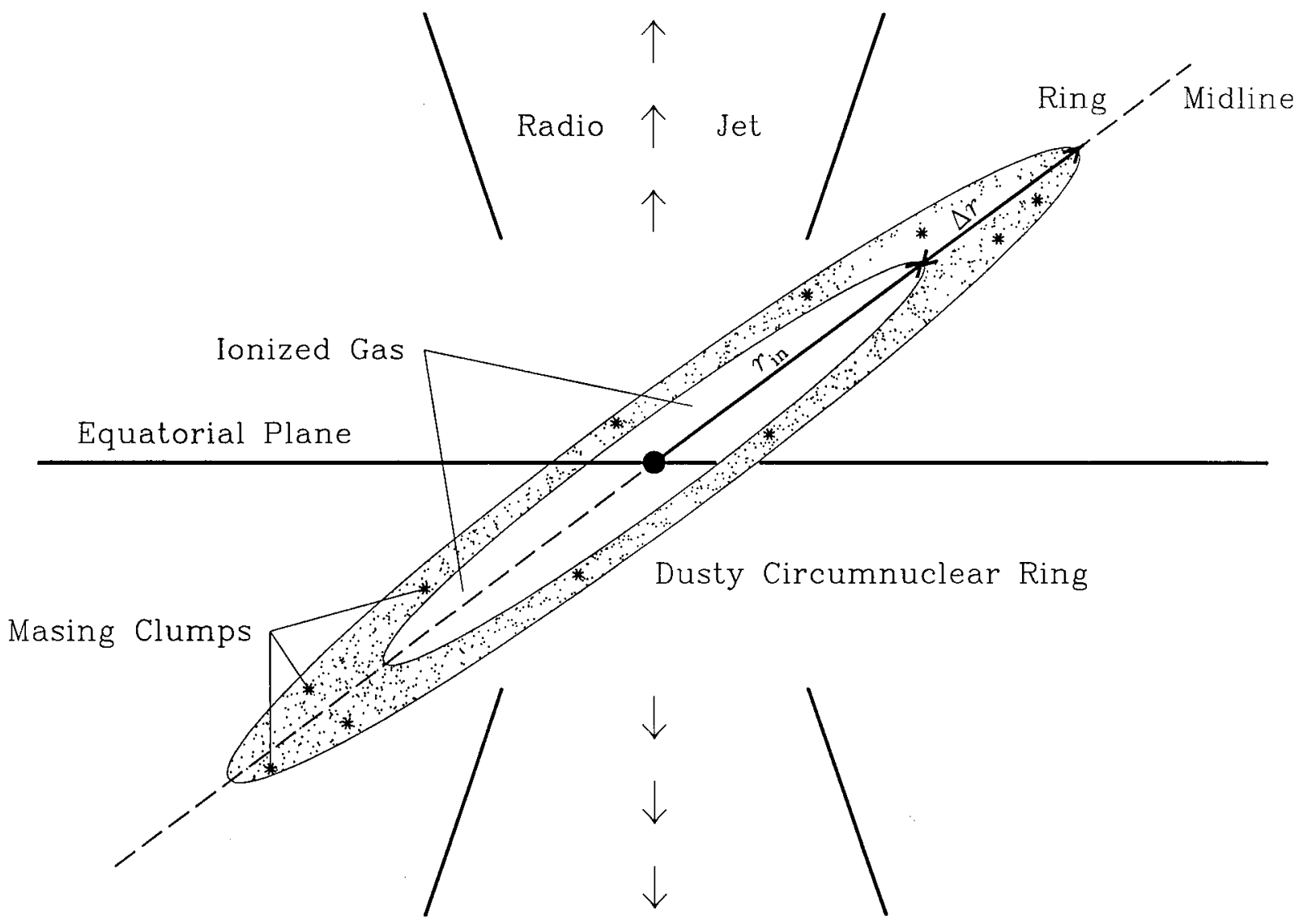

FIG. 2.-Schematic diagram of a clumped ring that orbits a black hole in an AGN. In an attempt to mimic the inferred conditions in NGC 1068, the ring plane is shown tilted at an angle of $45^{\circ}$ with respect to the equatorial plane, whereas the radio jet axis is depicted as being normal to that plane. For a similar reason, the ratio of the disk width to its inner radius was taken as $\Delta r / r_{\text {in }} \approx 0.6$. The ring rotation axis is shown at an inclination of $80^{\circ}$ with respect to the line of sight. The gas in the ring is assumed to be dusty (consistent with the fact that the dust sublimation radius lies interior to $r_{\text {in }}$ ) and to be composed of cold, dense clumps and possibly also a warm, more tenuous interclump medium. In the case of NGC 1068 there is evidence for ionized gas that produces free-free radio emission within $r_{\text {in }}$. This emission (which may in part originate in the inner rim of the ring) could be amplified into maser radiation by clumps located near $r_{\text {in }}$. High-velocity maser features may be associated with self-amplifying clumps situated along the ring midline.

required for our line of sight through the disk plane to intercept, on the average, two overlapping clouds within a velocity-coherence length, by setting $\mathscr{N}_{c, \text { min }} \approx 1 / \pi R_{c}^{2} \ell_{\text {coh }}$. Substituting from equation (7), we obtain

$$
\mathscr{N}_{c, \min } \approx 1.2 \times 10^{13}\left(\Delta v / 10^{-3} v_{\text {los }}\right)^{-1 / 2} R_{c 13}^{-2} r_{0.1}^{-1} \mathrm{pc}^{-3} .
$$

It is noteworthy that this fiducial value of the cloud space density, which is applicable to any scenario that interprets the high-velocity maser features in terms of overlapping clumps, is consistent with independent estimates for BELR clouds in typical AGNs (e.g., Arav et al. 1997, 1998).

The minimum cloud space density given by equation (17) corresponds to a cloud volume filling factor

$$
\begin{aligned}
f_{V, \text { min }} & \approx\left(2 R_{c} / a \ell_{\mathrm{coh}}\right) \\
& \approx 2.5 \times 10^{-4}\left(\Delta v / 10^{-3} v_{\mathrm{los}}\right)^{-1 / 2} a_{10}^{-1} r_{0.1}^{-1} R_{c 13}
\end{aligned}
$$

and a cloud contribution to the mean gas density in the disk of $\left\langle n_{c}\right\rangle \approx f_{V, \min } n_{c}$. Since the radial extent of the masing ring is typically $\gg \ell_{\text {coh }}$, one expects the clumped disk component to provide a nonnegligible shielding column in the direction of the central continuum. For a cloud located at a distance $\Delta r=r-r_{\text {in }}$ from the ring's inner edge, the shielding column of intervening clumps can be approximated by $N_{\text {cent }} \approx\left\langle n_{c}\right\rangle \Delta r \approx\left(6 v_{\text {los }} / \Delta v\right)^{1 / 2}(\Delta r / r) n_{c} R_{c} / a$. We can elimi- nate $R_{c}$ from this expression by fixing the effective maser emission measure (eq. [16]) at $\xi \approx 1$, the minimum value for efficient masing (EHM89). Then

$$
R_{c 13}=5 \xi \Delta v_{5} x_{-5}\left(\mathrm{H}_{2} \mathrm{O}\right) n_{c 9}^{-2},
$$

and, for a ring around a black hole of mass $M_{\mathrm{bh}}=10^{7} M_{7}$ $M_{\odot}$,

$$
\begin{aligned}
N_{\text {cent }} \approx & 1.6 \times 10^{23} \xi M_{7}^{1 / 4} r_{0.1}^{-1 / 4} \Delta v_{5}^{1 / 2} \\
& \times(\Delta r / 0.5 r) x_{-5}^{-1}\left(\mathrm{H}_{2} \mathrm{O}\right) n_{c 9}^{-1} a_{10}^{-1} \mathrm{~cm}^{-2} .
\end{aligned}
$$

We now combine the constraints given by the values of $N_{\min }, N_{\max }$, and $N_{\text {cent }}$. Using the parameters of NGC 1068 as an example, we choose a radius near $r_{\text {out }}(r=1 \mathrm{pc})$ and plot these three column densities in an $N_{\text {shield }}$ versus $x\left(\mathrm{H}_{2} \mathrm{O}\right)$ diagram (Fig. 3). The curves are labeled by the chosen cloud densities. For any particular value of $n_{c}$, we focus on the portion of the oblique $\left(N_{\text {cent }}\right)$ line that lies between the corresponding horizontal $\left(N_{\min }\right.$ and $\left.N_{\max }\right)$ lines. This segment of the $N_{\text {cent }}\left(n_{c}\right)$ curve corresponds to a certain range of water abundances $x\left(\mathrm{H}_{2} \mathrm{O}\right)$. Over that range, dusty clouds located between $r_{\text {in }} \approx 0.65 \mathrm{pc}$ and $r=1 \mathrm{pc}$ can provide sufficient shielding of the central continuum to render the cloud at $r=1$ pc molecular, but not so much shielding that the cloud's temperature drops below $\sim 250 \mathrm{~K}$. Since we chose a 


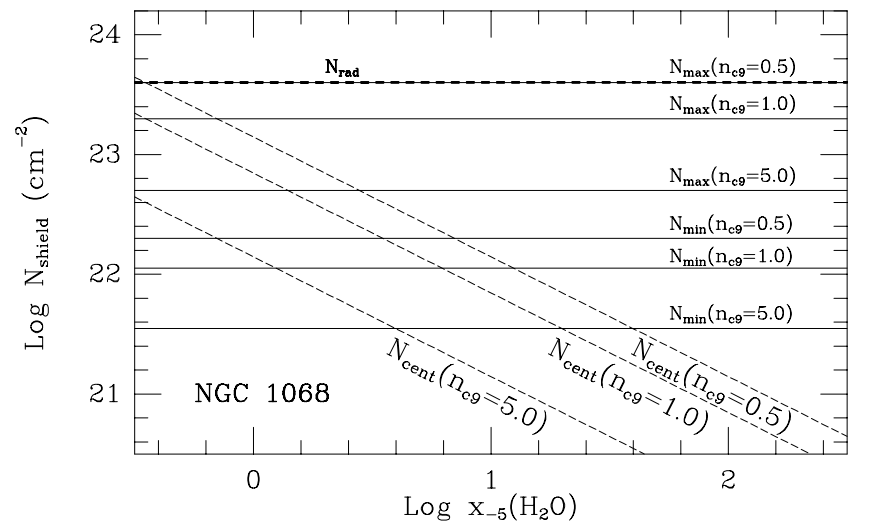

FIG. 3.-Phase-space diagram in the shielding column vs. fractional $\mathrm{H}_{2} \mathrm{O}$ abundance plane of the minimalist irradiated ring model for NGC 1068. The dusty gas column densities $N_{\min }, N_{\max }$, and $N_{\text {cent }}$ correspond, respectively, to the minimum shielding that is required for a cloud to be molecular (see Fig. 1), the maximum shielding that allows the temperature of a cloud to exceed $\sim 250 \mathrm{~K}$ so that collisional pumping can operate (which, in turn, determines the outer maser radius $r_{\text {out }}$ ), and the shielding of the central continuum by intervening clouds (see eq. [20]). All these columns depend on the cloud density $n_{c}$ and were calculated for a cloud located at $r=1 \mathrm{pc}$. In order for the model to be self-consistent, the water abundance must be such that $N_{\text {cent }}$ for the given cloud density lies between the corresponding values of $N_{\min }$ and $N_{\max }$. To guarantee that the ring is not dispersed by radiation pressure on the dusty gas, $N_{\max }$ should not be much smaller than $N_{\text {rad }}$ (eq. [21]).

radius near $r_{\max }$, we expect the cloud temperature to be close to $250 \mathrm{~K}$; the total shielding column must therefore be close to $N_{\max }$, but if $N_{\text {cent }}$ is lower than $N_{\max }$ one can attribute the difference to the presence of an intercloud medium. Since the requirement of a sufficiently high maser efficiency has already been incorporated into the expression for $N_{\text {cent }}$, the indicated range would represent a self-consistent masing ring model if the required water abundance could be attained.

Figure 4 shows the water abundance and temperature as a function of the distance $d$ from the irradiated surface of a dusty slab that is placed at the adopted fiducial distance $(r=1 \mathrm{pc})$ from the center of NGC $1068 .^{6}$ This figure demonstrates that abundances as high as $x_{-5}\left(\mathrm{H}_{2} \mathrm{O}\right) \approx 1$ are attained in the irradiated clouds (consistent with the values shown in Fig. 1 of Neufeld et al. 1994; note that Fig. 2 in that paper indicates significantly higher abundances, which we, however, were unable to reproduce). In conjunction with Figure 3, these results imply that essentially any cloud density that lies in the optimal masing range could be accommodated by a ring model that satisfies the indicated constraints. In particular, the requirement $N_{\text {cent }}\left[n_{c}\right.$, $\left.x\left(\mathrm{H}_{2} \mathrm{O}\right)\right]<N_{\max }\left(n_{c}\right)$ is evidently satisfied for $x_{-5}\left(\mathrm{H}_{2} \mathrm{O}\right) \approx 1$ irrespective of the value of $n_{c}$. (The insensitivity to the cloud density can be understood from the fact that $N_{\max }$ and $N_{\text {cent }}$ have a similar dependence on $n_{c}$.)

With all other parameters being equal, lower density clouds give rise to a higher shielding column $N_{\text {cent }}$ to the center. In a high-luminosity source like NGC 1068, a relevant additional constraint is the minimum column $N_{\text {rad }}$ of

${ }^{6}$ Note that dust-reprocessed radiation emitted within the slab is included in the calculation of the gas thermal structure. The contribution of this component is, however, expected to be reduced in a realistic clumpy disk. The opposite limit, in which the dust-reprocessed radiation is neglected altogether, was adopted in the derivation of $N_{\max }$ in $\S 3$.
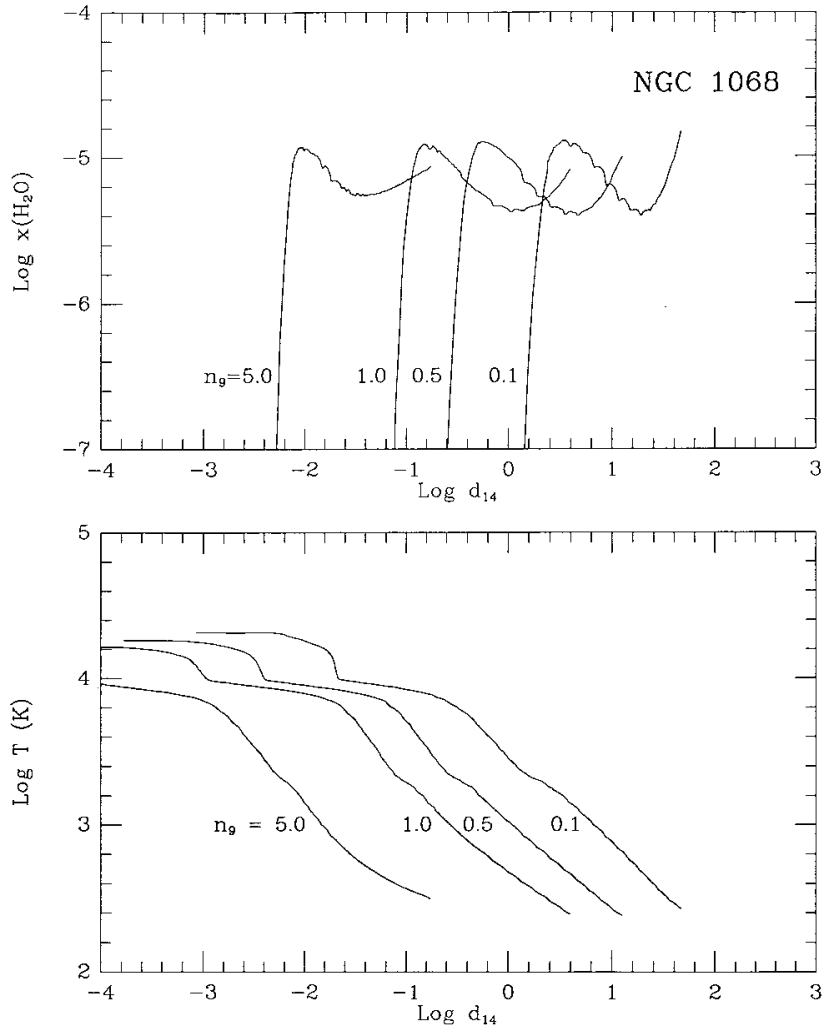

FIG. 4.-Fractional water abundance (top panel) and gas temperature (bottom panel) as a function of distance $d=10^{14} d_{14} \mathrm{~cm}$ into a dusty, uniform-density slab that is irradiated by the nuclear continuum of NGC 1068 (assumed to be unattenuated before it reaches the slab) at a distance of $1 \mathrm{pc}$ from the center. The curves are labeled by the value of the slab density $n_{\mathrm{H}}=10^{9} n_{9} \mathrm{~cm}^{-3}$ and terminate at the point where the temperature declines to $250 \mathrm{~K}$.

dusty gas that would prevent the ring from being dispersed by the radiation pressure force exerted by the central continuum. Since the effective Eddington luminosity for dust opacity is $L_{\text {crit }} \approx 10^{42} M_{7}$ ergs s$^{-1}$ (e.g., KK94), and since the bulk of the continuum radiation is absorbed within a dusty column of $\sim 10^{21} \mathrm{~cm}^{-2}$, we estimate

$$
N_{\mathrm{rad}} \approx 10^{23}\left(L_{\mathrm{bol}} / 10^{44} \mathrm{ergs} \mathrm{s}^{-1}\right) M_{7}^{-1} \mathrm{~cm}^{-2} .
$$

The value of $N_{\text {rad }}$ for NGC 1068 is indicated in Figure 3 by the heavy dashed line. If the typical cloud densities are $n_{c 9} \lesssim 0.5$, then $N_{\max } \gtrsim N_{\text {rad }}$ and the gas in the maser emission region can withstand ejection by the radiation pressure force. We note from equations (6) and (19) that the condition $R_{c}<w_{\text {coh }}$ implies, for $x_{-5}\left(\mathrm{H}_{2} \mathrm{O}\right) \lesssim 1$ and $\xi \gtrsim 1$, that a cloud at $r=1 \mathrm{pc}$ in this source must have $n_{c 9}>0.07$. Clouds with $n_{c 9} \approx 0.5$ would have $R_{c} \approx 2 \times 10^{14} \mathrm{~cm}$ and, even with $a \approx 10$, would still satisfy $\ell_{c}=a R_{c} \ll \ell_{\text {coh }}$ $\left(\approx 2 \times 10^{17} \mathrm{~cm}\right)$. In any case, we expect the disk to also contain nonmasing gas and therefore to have an even greater inertia against radiative acceleration. One indication of the presence of nonmasing gas in masing AGN disks is provided by the appearance of an absorption trough at the systemic velocity in the maser spectra of sources like NGC 4258 (see § 2.2). In the current model, this absorption can arise in irradiated gas beyond $r_{\text {out }}$, whose temperature lies in the range $\sim 100-250 \mathrm{~K}$. In the case of NGC 1068 it appears, making a rough extrapolation of the curves in Figure 4, that an absorbing column $\gtrsim N_{\text {rad }}$ could be main- 
tained at such temperatures even if the typical cloud densities are such that the total column $N_{\max }\left(n_{c}\right)$ in the masing region remains smaller than $N_{\text {rad }}$. The absorption optical depth is maximized at $T \approx 200 \mathrm{~K}$, and, for that temperature and $\Delta v_{5} \approx 1$, it is given by

$$
\tau_{\text {absorb }} \approx 5.9 \times 10^{-25} x_{-5}\left(\mathrm{H}_{2} \mathrm{O}\right) N_{\text {absorb }},
$$

where $N_{\text {absorb }}$ is the absorbing hydrogen nuclei column (e.g., MM98). By setting $N_{\text {absorb }} \gtrsim N_{\text {rad }}\left(\approx 2.4 \times 10^{23} \mathrm{~cm}^{-2}\right.$ for NGC 1068) and adopting $x_{-5}\left(\mathrm{H}_{2} \mathrm{O}\right) \approx 1$, we infer from equation (22) a systemic velocity flux reduction factor $\gtrsim 1.2$, which seems to be consistent with the data (see, e.g., Fig. 1 in Greenhill et al. 1996). Note, however, that the extent to which the observer's line of sight to the center intersects the $r \gtrsim r_{\max }$ absorbing region in any given source depends on the disk geometry and the viewing angle.

The properties of the masing ring in NGC 1068 that are implied by this interpretation bear a remarkable resemblance to the inferred characteristics of the CND in the Galactic center. In NGC 1068 , adopting $n_{c 9} \approx 0.5$ as a typical cloud density, we deduce $f_{V \text {, } \min } \approx 3 \times 10^{-4}$ (eq. [18]) and a lower limit on the mass (considering only the masing gas between $r_{\text {in }}$ and $r_{\text {out }}$ and adopting a disk halfthickness of $\sim 0.1 r$ ) of $\sim 4 \times 10^{3} M_{\odot}$. Just like the NGC 1068 maser emission region, the Galactic CND has the appearance of a ring with a sharp inner edge (located at a comparable distance from the center, $r_{\text {in }} \approx 1.5 \mathrm{pc}$; the outer radius of the CND has been inferred to be $\gtrsim 7 \mathrm{pc}$ ): it is inclined with respect to the galactic plane (by $\sim 20^{\circ}-25^{\circ}$ ) and is slightly warped (tilt angle varying in the range $\sim 10^{\circ}-$ $25^{\circ}$; Güsten et al. 1987), and it exhibits a dominant circular motion (which in the case of the CND corresponds to a nearly uniform rotational speed of $\sim 110 \mathrm{~km} \mathrm{~s}^{-1}$ ) but with a nonnegligible velocity dispersion. It also has a similar inferred mass $\left(\sim 10^{4} M_{\odot}\right.$ within $\left.4 \mathrm{pc}\right)$ and an indicated central black hole of comparable mass (within a factor of $\sim 6$; see Genzel et al. 1997). It is particularly noteworthy that the CND appears to be highly clumped: HCN observations (e.g., Jackson et al. 1993) have revealed that the bulk of the material is characterized by hydrogen nuclei densities in the range $\sim 10^{6}-10^{8} \mathrm{~cm}^{-3}$ and volume filling factors $\sim 10^{-3}-10^{-2}$, corresponding to mean densities in the range $\sim 10^{4}-10^{5} \mathrm{~cm}^{-3}$, and by an area covering factor $\sim 0.1$. In fact, there is no observational evidence for the presence of a substantial component of a low-density interclump medium in the CND. Also, in both cases, there is evidence of mass inflow into the ring, which on larger $\left(\sim 10^{2}-10^{3} \mathrm{pc}\right)$ scales is associated with bar streaming (e.g., Helfer \& Blitz 1995). In fact, as was first noticed by Greenhill \& Gwinn (1997), the position angles of the maser ring axis and the nuclear $\mathrm{CO}$ bar in NGC 1068 differ by only $\sim 20^{\circ}$. On the basis of these similarities one can surmise that the Galactic CND would appear as a water maser ring akin to that in NGC 1068 if it had a comparably bright high-energy central continuum. ${ }^{7}$ The high-luminosity nonthermal emission in NGC 1068, which is a signature of the currently active phase of its nucleus, is evidently powered by accretion into the center. As was pointed out by Helfer \& Blitz (1995), the main difference in the circumnuclear molecular gas distribution

\footnotetext{
${ }^{7}$ Although one $\mathrm{H}_{2} \mathrm{O}$ maser was detected near the inner edge of the CND (Levine et al. 1995), it appears to coincide with a luminous, reddened star and is therefore likely to have a different origin than the NGC 1068 megamasers.
}

between NGC 1068 and the Milky Way is that the former appears to have a large reservoir of gas (which could be the source of fuel to the central engine) in the inner spiral arms, whereas in our own galaxy there is a relative paucity of gas on similar scales.

The origin of the Galactic CND is still an open question, and if similar structures indeed manifest themselves as extragalactic megamaser disks, then its resolution could provide important clues to the nature of accretion in AGNs (see $\S 6$ ). One of the key issues is the presence of a welldefined inner edge at such a comparatively large distance from the center; interestingly, the existence of such an edge has been indicated also in the $\mathrm{H}_{2} \mathrm{O}$ maser distributions of NGC 1068 and NGC 4258. If the inflowing gas in the disk is broken up into distinct clumps as it approaches $r_{\text {in }}$, then the accretion efficiency could conceivably decrease at lower radii, providing at least a partial explanation for the occurrence of an "edge" at that location. However, it appears that mass leaves the inner edge of the CND in the form of "streamers" at about the same rate as it is inferred to flow through the disk (Jackson et al. 1993). It has been suggested that the presence of clumps and the large measured velocity dispersion in the CND can be attributed to tidal disruption (e.g., Genzel et al. 1994). The Roche critical density, given (for an assumed molecular weight of 2.33) by

$$
n_{\mathrm{H}, \mathrm{crit}} \approx 3.5 \times 10^{11} M_{7} r_{0.1}^{-3} \mathrm{~cm}^{-3}
$$

is $\sim 2 \times 10^{9} \mathrm{~cm}^{-3}$ at the inner edge of the maser ring in NGC 1068. If $n_{c}$ exceeds this value then the masing clouds in this object could withstand tidal disruption and might therefore be relatively long lived.

The maser features that delineate the inner edge of the disk in NGC 1068 likely amplify a background radio continuum source, as has been inferred to be the case for the systemic masers in NGC 4258 (see § 2.2). In fact, Gallimore et al. (1997) obtained radio images of a parsec-scale disk in NGC 1068 that they interpreted in terms of a hot, ionized gas at the inner edge of the nuclear disk. It is therefore possible that the ring inner edge itself is the source of the background radiation. It is interesting to note in this connection that the Galactic CND also exhibits an ionized, radio-emitting inner rim (e.g., Genzel et al. 1994). In fact, only part of the CND's inner circumference (notably, the so-called Western Arc) is manifested in this fashion, which has been attributed to absorption of the central ionizing radiation in certain directions by infalling matter within the central cavity (Jackson et al. 1993). A similar explanation may perhaps be relevant to the fact that only one quadrant of the inner rim of the NGC 1068 ring has a measurable maser flux. The confinement of the maser emission to the vicinity of the disk inner edge could reflect the fact that the background radiation is intercepted and absorbed within a narrow radial range near $r_{\text {in }}$. It is nevertheless puzzling why one does not observe enhanced maser emission near the center line. In the case of a planar disk and a $90^{\circ}$ viewing angle, both background and self-amplification by clouds are expected to contribute to the flux near the systemic velocity on account of the comparatively large velocity-coherence length near the center line. (As noted above, the emission originating right along and in the immediate vicinity of the center line would be subject to absorption by the nonmasing gas at $r>r_{\text {out }}$.) The absence of a flux peak near the systemic velocity may be due to the disk's departure from planarity or velocity coherence or to the viewing angle 
being sufficiently smaller than $90^{\circ}$. It is, however, also conceivable that the total shielding column through the disk is large enough for all the ionizing continuum radiation to be absorbed near $r_{\text {in }}$ (see MM98). In that case a continuumirradiation excitation of the high-velocity features would require raising the masing gas above the midplane as originally envisioned by Neufeld \& Maloney (1995).

Before concluding our discussion of this scenario it is of interest to inquire whether the minimalist model that we considered for NGC 1068 could also be applied to NGC 4258. Figures 5 and 6 are the analogs of Figures 3 and 4, respectively, and are based on the same assumptions, except

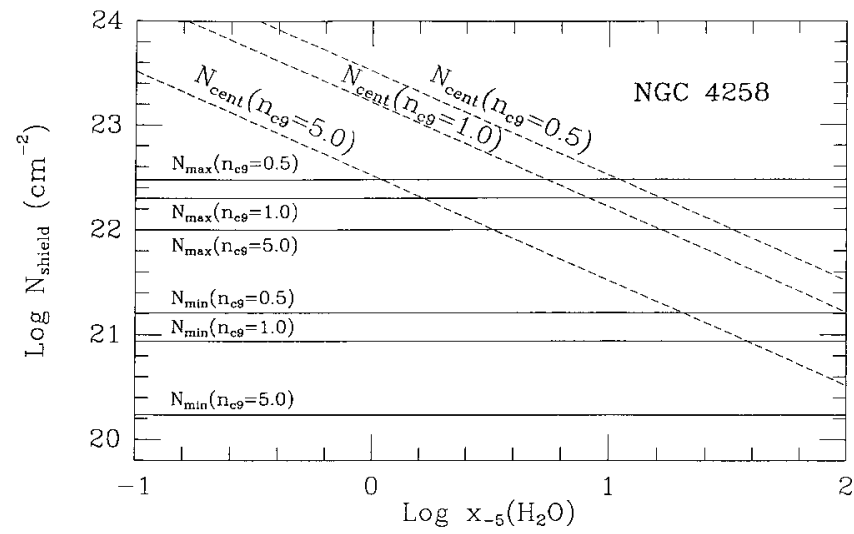

Fig. 5. - Same as Fig. 3, but for the continuum source parameters of NGC 4258 and $r=0.25$ pc. Because of the comparatively low luminosity of this source, the shielding column $N_{\text {rad }}$ (eq. [21]) is not a relevant constraint in this case.
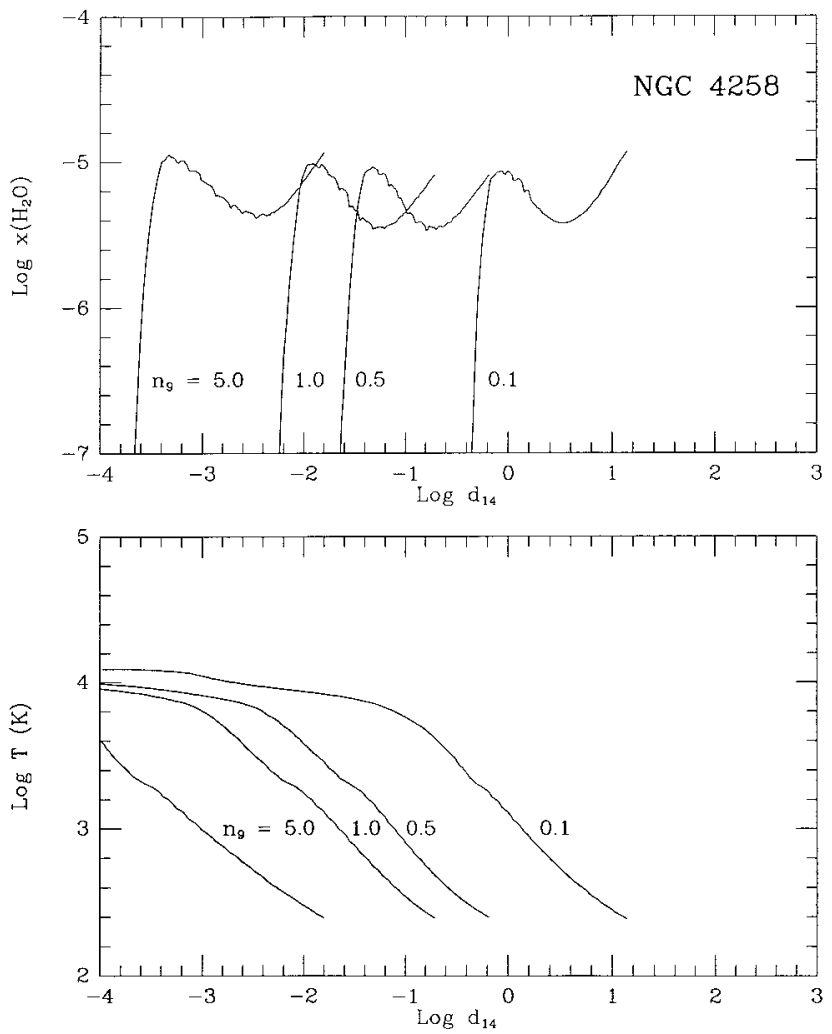

FIG. 6.-Same as Fig. 4, but for the continuum source parameters of NGC 4258 and $r=0.25 \mathrm{pc}$. that in this case we use the inferred spectrum of NGC 4258 and set $r=0.25 \mathrm{pc}$ (which is close to $r_{\text {out }}$ in this source). We do not plot the value of $N_{\text {rad }}$ (eq. [21]) since radiation pressure acceleration is not an issue in this low-luminosity $\left(L_{\mathrm{bol}} \approx 10^{42}\right.$ ergs $\left.\mathrm{s}^{-1}\right)$ object. We find that, as before, the photoionization-induced water abundance does not rise above $x_{-5}\left(\mathrm{H}_{2} \mathrm{O}\right) \approx 1$ and that, for this value, $N_{\text {cent }}\left(n_{c}\right)$ is significantly larger than $N_{\max }\left(n_{c}\right)$. In other words, for the minimum cloud space density (eq. [17]) required to explain the high-velocity features in terms of overlapping maser clumps, the implied cloud self-shielding of the central continuum is too high to allow the masing region to extend as far as it is observed to do.

A possible resolution of the above difficulty is that $x\left(\mathrm{H}_{2} \mathrm{O}\right)$ is, in fact, greater than $\sim 10^{-5}$. One potential source of water in dusty clouds is the evaporation of icy mantles of grains that become exposed to the central radiation field and are heated to a temperature of $\gtrsim 100 \mathrm{~K}$. Ice could form on the surfaces of grains that are embedded in a cold, molecular disk through the hydrogenation of oxygen (Brown, Charnley, \& Millar 1988). Infrared observations of star-forming regions in dense molecular clouds have revealed that $\sim 10 \%$ of the elemental oxygen is in water ice: in that case $x\left(\mathrm{H}_{2} \mathrm{O}\right)$ could be $\sim 6 \times 10^{-5}$ (Ceccarelli, Hollenbach, \& Tielens 1996). Brown et al. (1988) originally hypothesized that this mechanism could yield water abundances as high as $\sim 5 \times 10^{-4}$. For $x_{-5}\left(\mathrm{H}_{2} \mathrm{O}\right) \approx 6$, Figure 5 indicates that a planar disk model could be self-consistent only for $n_{c 9}>1$. For cloud densities of that order one can infer from Figure 6 that the column of absorbing gas at $r>r_{\max }$ would be $\lesssim 10^{24} \mathrm{~cm}^{-2}$, which, by equation (22) with $x_{-5}\left(\mathrm{H}_{2} \mathrm{O}\right) \approx 6$, would yield a flux reduction factor $\sim 30$. This is consistent with the systemic velocity value of $\sim 10$ measured in this source. For $\xi \approx 1, x_{-5}\left(\mathrm{H}_{2} \mathrm{O}\right) \approx 6$, and $n_{c 9} \gtrsim 1$, the characteristic cloud radius would be $r_{c 13} \lesssim 1$ (eq. [19]). We conclude that if ice evaporation as invoked in collapsing protostellar envelope calculations is also a viable mechanism in molecular accretion flows in AGNs, then the minimalist planar ring model could, in principle, also apply to a source like NGC 4258. If the mantle evaporation mechanism is for some reason inapplicable, then NGC 4258 might still represent a clumpy ring, but in order for the maser emission to be excited by continuum irradiation the maser clouds could not all lie in the same plane.

\section{CLOUDS UPLIFTED BY A DISK-DRIVEN WIND}

In $\S 4$ we discussed irradiated clumpy rings and showed that, while they may adequately explain the maser distributions in objects like NGC 1068 and perhaps even NGC 4258 , certain aspects of the observations may be easier to interpret if the masing clouds are not strictly coplanar. Since the maser disks in both of these sources in fact appear to be slightly warped (a feature that they share also with the Galactic circumnuclear ring), one is led to examine plausible physical mechanisms that could give rise to a noncoplanar distribution of masing clouds. As we noted in $\S 1$, previously proposed warping scenarios were constructed for homogeneous disk models. Although these mechanisms would presumably remain applicable if the disk contained a turbulent velocity field, it is less clear how efficiently they would operate in a highly inhomogeneous disk with a distinct, low-filling-factor cloud component. In this section we address the issue of a noncoplanar cloud distribution and 
propose an alternative to the warped disk interpretation of continuum-irradiated megamaser sources. We suggest that the maser features represent diamagnetic molecular clouds that are uplifted from the surface of a circumnuclear accretion disk by a dusty hydromagnetic wind. In this picture the wind is driven centrifugally along rotating magnetic field lines that are anchored in the disk (Blandford \& Payne 1982). Dust from the outer, molecular regions of the disk is carried out with the gas and survives destruction by the intense central continuum along wind streamlines whose footpoints in the disk lie beyond the dust sublimation radius $r_{\text {sub }}$. KK94 showed that such a wind could produce the obscuration of the central continuum source that has been attributed to the phenomenological molecular "torus" that is inferred to surround the BELR of Seyfert 2 galaxies (e.g., Antonucci 1993). The incorporation of clouds into such a wind has previously been considered in the context of models of BELR clouds in Seyfert galaxies and QSOs (Emmering, Blandford, \& Shlosman 1992; Bottorff et al. 1997), EUV and X-ray absorption-line clouds in BL Lacertae objects (Königl et al. 1995; Kartje et al. 1997), and broad absorption line QSOs (de Kool \& Begelman 1995; Kartje \& Königl 1997, 1999). Although in this paper we do not specifically address the origin of the clouds, the internal densities and space densities of the masing clumps that we model are consistent with the parameters inferred for BELR clouds (e.g., Baldwin et al. 1995; Arav et al. 1997, 1998).

In applying the uplifted-cloud scenario to $\mathrm{H}_{2} \mathrm{O}$ megamaser sources we show that the necessary shielding from the high-energy central continuum photons can be provided for comparatively massive clouds that initially move on nearly circular trajectories close to the disk surface. Since the velocity field of these clouds is well approximated by the rotation law of the underlying disk, it follows from the considerations of $\S 2$ that this scenario can account for the detection of $\mathrm{H}_{2} \mathrm{O}$ maser features in Seyfert galaxies that are viewed at large angles to their symmetry axes. In this picture the apparent warp in the maser distribution may result from an observational selection effect: a cloud uplifted at a given radius would attain optimal masing conditions at a height large enough that shielding by the dust in the intervening wind and clouds is not too high, but not so large that the shielding column or the cloud density become too low for efficient masing. Both the shielding column to the center and the cloud density (which is determined by the confining ambient pressure) drop rapidly with height in centrifugally driven outflows of the type that we envision. Therefore, for typical wind mass outflow rates, the maser emission region would be confined to the immediate vicinity of the disk surface. Since the maser-shielding column in this interpretation is directly related to the equivalent $\mathrm{X}$ ray-absorbing hydrogen column density, both being associated with the disk-driven wind (see KK94), this model also readily accounts for the inferred correlation between the probability of detecting $\mathrm{H}_{2} \mathrm{O}$ maser emission in a Seyfert galaxy and the magnitude of its X-ray-absorbing column (Braatz et al. 1997).

\subsection{Cloud Dynamics}

In our proposed picture, dense clouds fragmented from the disk are uplifted by the wind ram pressure and transported along the streamlines (with radiation pressure possibly contributing to the acceleration once the clouds get exposed to the central continuum source; see Kartje \&
Königl 1997, 1999). A nominal estimate of the maximum cloud mass that can be ejected from the disk at a radius $r$ may be obtained by balancing the wind ram pressure and tidal gravitational forces (see eq. 25]). This gives

$$
\begin{aligned}
M_{c, \max } & \approx \pi \dot{M}_{w} R_{c}^{2} /\left(G M_{\mathrm{bh}} r\right)^{1 / 2} \\
& =9.8 \times 10^{23} \dot{M}_{w,-3} R_{c 13}^{2} M_{7}^{-1 / 2} r_{0.1}^{-1 / 2} \mathrm{~g},
\end{aligned}
$$

where $\dot{M}_{w}=10^{-3} \dot{M}_{w,-3} M_{\odot} \mathrm{yr}^{-1}$ is the mass outflow rate in the wind. The clouds can be assumed to be pressure confined by the wind magnetic field. The approximate cloud trajectories under the joint action of the wind-cloud drag force and the gravitational field of the central mass can be obtained by solving the equation

$$
\frac{d v_{c}}{d t} \approx-\frac{G M}{r^{2}} \hat{r}+\frac{\pi R_{c}^{2} \rho_{w}}{M_{c}}\left|v_{w}-v_{c}\right|\left(v_{w}-v_{c}\right),
$$

where $v_{c}, M_{c}, v_{w}$, and $\rho_{w}$ are the cloud velocity, cloud mass, wind velocity, and wind mass density, respectively. We assume that the cloud density is determined by the local wind magnetic field strength $B_{w}\left(n_{c} \propto B_{w}^{2}\right.$ for $T$ nearly constant). We further assume that the mass of each cloud does not change and that the clouds maintain a roughly spherical shape (i.e., $R_{c} \propto B_{w}^{-2 / 3}$ ).

In view of the minimum shielding required for keeping the clouds molecular (see Fig. 1), the masing clumps must be located at $r>r_{\text {sub }}$ behind a sufficiently large column of dusty wind and uplifted clouds. The precise geometry and kinematic properties of the maser emission region depend both on the cloud characteristics (sizes, masses, and ejection radii) and on the dynamical properties of the wind. In particular, the wind mass outflow rate $\dot{M}_{w}$ is a key parameter. In sources with a high value of $\dot{M}_{w}$ the dust shielding might be effective up to comparatively high latitudes and the projected locus of efficient maser emission within the wind would take the form of a narrow strip (whose width increases with height above the disk) that extends along the innermost dusty streamline (see Fig. 7a). In this case the line-of-sight velocities of high-latitude maser features would be expected to drop with distance from the rotation axis in a sub-Keplerian fashion even if the equatorial disk were Keplerian (see Fig. 7b). By contrast, in winds with a lower mass outflow rate, which are less effective at shielding the central continuum, the projected locus of efficient masing would be a narrow strip extending along (and right on top of) the disk surface (Fig. 7a). To ensure that their trajectories lie within the most highly shielded wind region close to the disk surface, the masing clouds in this case would need to be fairly heavy $\left(M_{c}>M_{c, \text { max }}\right.$; see eq. [24]). The motions of such relatively massive clouds would closely mirror the ambient velocity field near the disk surface, and hence, in the case of a Keplerian disk, their line-of-sight velocities would satisfy $v_{\text {los }} \propto r^{-1 / 2}$ (Fig. $7 b$ ). The latter alternative is evidently the one that best describes the basic morphology and kinematics of the $\mathrm{H}_{2} \mathrm{O}$ masers in a source like NGC 4258. In light of the arguments presented in $\S 2$, winduplifted clouds that overlap along the line of sight could, in principle, account for the observed flux level and variability of the maser features in this source.

\subsection{A Clumpy Disk-driven Wind Model}

To flesh out the above scenario we now incorporate the dynamical constraints on the uplifted maser clouds into a 

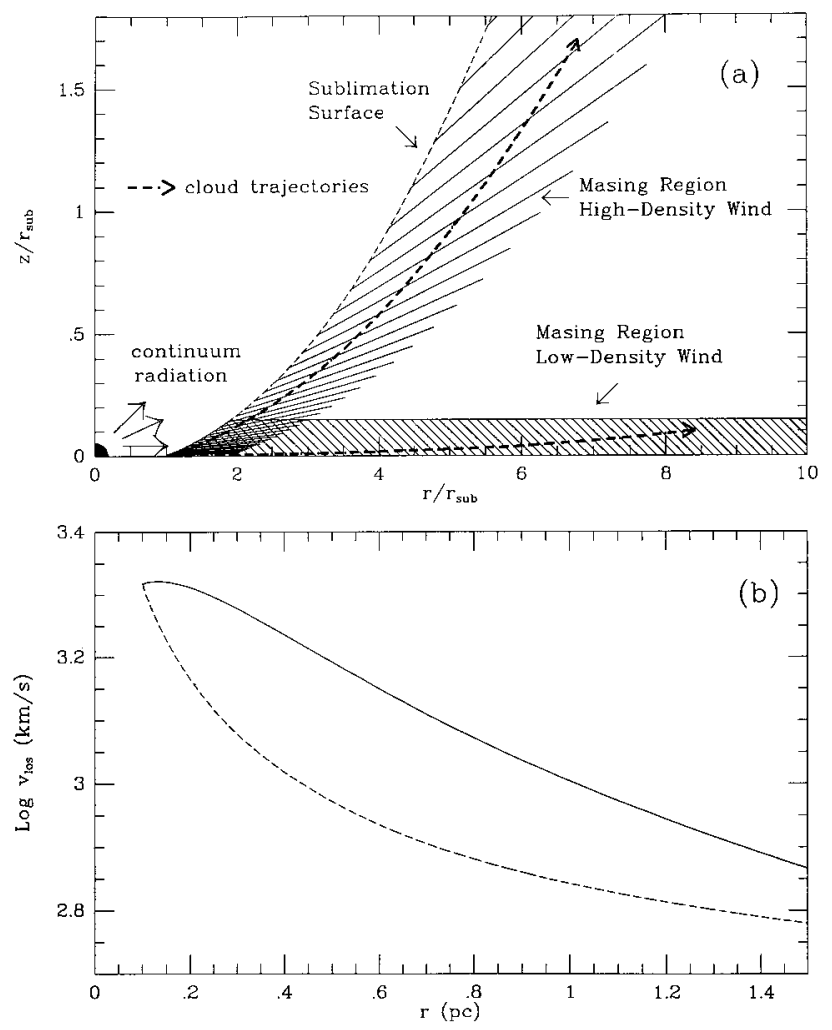

FIG. 7.- (a) Schematic diagram illustrating the expected regions of efficient maser emission from clouds embedded in accretion disk-driven hydromagnetic winds that are viewed orthogonally to the rotation axis. The light dashed curve indicates the dust sublimation surface in the wind, while the shading demarcates the masing zones: along the sublimation surface for high-density winds and along the disk surface for lower density winds. The heavy dashed curves show poloidal projections of representative cloud trajectories. Lighter clouds (of mass $M_{c} \lesssim M_{c, \max }$; see eq. [24]) tend to move along wind streamlines and rise to high altitudes, whereas heavier clouds $\left(M_{c} \gtrsim M_{c, \max }\right)$ tend to move across wind streamlines and remain close to the disk surface. The maser features in a source like NGC 4258 are evidently best modeled by a comparatively low density wind that gives rise to a narrow efficient masing strip near the disk surface. $(b)$ Lineof-sight velocity as a function of (cylindrical) radius for clouds moving in a disk-driven wind. The wind is assumed to be radially self-similar and is described by model A1 in KK94 (with a total mass outflow rate of $0.03 M_{\odot}$ $\mathrm{yr}^{-1}$ and a central mass $\left.M_{\mathrm{bh}}=3.5 \times 10^{7} M_{\odot}\right)$. The solid and dashed curves represent clouds with masses $M_{c} \approx 0.5 M_{c, \text { max }}$ and $M_{c} \approx 11 M_{c, \max }$, respectively. The latter curve adequately describes the nearly Keplerian velocity field of the maser features in a source like NGC 4258.

"generic" model of a disk-driven hydromagnetic wind. As we discussed in $\S 5.1$, the maser observations in an object like NGC 4258 are best described in the context of this picture in terms of clouds of masses $M_{c}=$ $(4 \pi / 3)\left(1.4 m_{\mathrm{H}}\right) R_{c}^{3} n_{c} \gtrsim 10 M_{c, \max }$ (where the factor 1.4 accounts for the assumed composition of molecular hydrogen with a $20 \%$ helium abundance). We thus parameterize $M_{c}=\eta M_{c, \max }$, with $\eta=10 \eta_{10}$. Another relation between the cloud and wind properties is provided by the pressure confinement condition. We assume that the clouds are diamagnetic (i.e., that they are not penetrated by the ambient magnetic field) and impose a balance between their internal (mostly thermal) pressure and the wind (mostly magnetic) pressure. This can be written as

$$
\frac{B_{w}^{2}}{8 \pi} \approx 0.6 n_{c} k T_{c},
$$

(where the factor 0.6 accounts for the assumed composition).

To make the model self-contained, we assume that the wind extracts most of the angular momentum of the accreted matter. This allows us to relate the magnetic field strength in the wind to the mass accretion rate $\dot{M}_{\text {acc }}$ through the disk. Using the angular momentum conservation relation for a thin, equatorial disk, $\dot{M}_{\mathrm{acc}} v_{\mathrm{K}} / 8 \pi r^{2} \approx$ $B_{z}\left|B_{\phi, s}\right| / 4 \pi$, (where $B_{z}$ and $B_{\phi, s}$ are, respectively, the vertical component and the azimuthal surface component of the disk magnetic field), we obtain

$$
\frac{B_{w}^{2}}{8 \pi}=\frac{\dot{M}_{\mathrm{acc}} v_{\mathrm{K}}}{16 \pi \beta r^{2}},
$$

where the parameter $\beta=B_{z}\left|B_{\phi, s}\right| / B_{w}^{2}$ depends on the detailed magnetic field configuration near the disk surface. In the cold, radially self-similar wind model of Blandford \& Payne (1982), which has been employed in several of the applications cited at the beginning of this section, one can express $\beta$ in terms of the self-similarity variables $\kappa$ (the normalized mass-to-flux ratio), $\lambda$ (the normalized total specific angular momentum), and $\xi_{0}^{\prime}$ (the radial-to-vertical field component ratio at the disk surface) as $\beta=\kappa(\lambda-1) /\left[\kappa^{2}(\lambda\right.$ $\left.-1)^{2}+\left(1+\xi_{0}^{\prime 2}\right)\right]$. This yields $\beta \approx 0.32$ for the wind solution used in Figure 7 (see Table 1 in KK94). We thus parameterize $\beta=0.3 \beta_{0.3}$.

To complete the specification of the model we need to relate the wind mass outflow rate (which appears in eq. [24] for $M_{c, \max }$ ) to the disk mass accretion rate (which appears in eq. [27]). We parameterize $\dot{M}_{w}=\delta \dot{M}_{\text {acc }}$ and choose $\delta_{0.1}=$ $\delta / 10$ as a representative normalization factor. Using the adopted fiducial values of $\beta, \eta$, and $\delta$, and parameterizing the temperature in the masing clouds by $T_{c 500}=\left(T_{c} / 500 \mathrm{~K}\right)$, we can combine the above expressions to get

$$
\begin{aligned}
R_{c} & \approx \frac{36 \pi \beta \eta \delta k T_{c} r^{2}}{7 G M_{\mathrm{bh}} m_{\mathrm{H}}} \\
& \approx 1.4 \times 10^{13} \beta_{0.3} \eta_{10} \delta_{0.1} T_{c 500} r_{0.1}^{2} M_{7}^{-1} \mathrm{~cm} .
\end{aligned}
$$

It is remarkable that the typical cloud radius obtained in this fashion for a source like NGC 4258 agrees with the value inferred from the requirements of efficient maser pumping (see eq. [19]). If we explicitly impose the condition that the maser efficiency factor $\xi$ (eq. [16]) be $\gtrsim 1$, we can directly estimate the characteristic mass accretion rate through the disk,

$$
\begin{aligned}
\dot{M}_{\mathrm{acc}} \approx & 2.7 \times 10^{-2} \beta_{0.3}^{1 / 2} \eta_{10}^{-1 / 2} \delta_{0.1}^{-1 / 2} \xi^{1 / 2} \\
& \times \Delta v_{5}^{1 / 2} x_{-5}\left(\mathrm{H}_{2} \mathrm{O}\right)^{-1 / 2} T_{c 500}^{1 / 2} r_{0.1}^{3 / 2} M_{\odot} \mathrm{yr}^{-1} .
\end{aligned}
$$

Interestingly, this value is compatible with the ADAF interpretation of the NGC 4258 spectrum (see $\S 1$ ). ${ }^{8}$ In conjunction with equation (27) we then deduce

$$
\begin{aligned}
B_{w} \approx 4.4 & \times 10^{-2} \beta_{0.3}^{-1 / 4} \eta_{10}^{-1 / 4} \delta_{0.1}^{-1 / 4} \xi^{1 / 4} \\
& \times \Delta v_{5}^{1 / 4} x_{-5}\left(\mathrm{H}_{2} \mathrm{O}\right)^{-1 / 4} T_{c 500}^{1 / 4} M_{7}^{1 / 4} r_{0.1}^{-1 / 2} \mathrm{G} .
\end{aligned}
$$

The toroidal field component $\left|B_{\phi, s}\right|$ would be smaller than this value; using again the Blandford \& Payne (1982)

\footnotetext{
${ }^{8}$ As we show in Appendix A, if a centrifugally driven wind carries away much of the disk angular momentum, then the ADAF interpretation can also be compatible with the radiation-induced warping instability model for this source.
} 
similarity variables, we deduce $\left|B_{\phi, s}\right| / B_{w}=\kappa(\lambda-1) /\left[\kappa^{2}(\lambda\right.$ $\left.-1)^{2}+\left(1+\xi_{0}^{\prime 2}\right)\right]^{1 / 2}$, which for the wind solution used in Figure 7 is $\sim 0.55$. Hence, at a distance $r_{0.1} \approx 2$ in NGC 4258 (where $M_{7} \approx 3.5$ ), a line-of-sight field component of $\sim 2 \times 10^{-2} \mathrm{G}$ is predicted in the wind. This is lower than the upper limit of $0.3 \mathrm{G}$ inferred from circular polarization measurements in the maser feature at that location (Herrnstein et al. 1998b), although we note that, under our assumption of diamagnetic clouds, the magnetic field amplitude in the masing clumps might in fact be lower than the estimate (eq. [30]) for the homogeneous outflow component.

As a self-consistency check, we evaluate the shielding column associated with the dusty wind itself. Assuming that the wind is atomic and has a vertical speed $v_{w, z}=\chi C\left(T_{w}\right)$, where $\chi=3 \chi_{3}$ and $C\left(T_{w}\right)$ is the isothermal speed of sound corresponding to the wind temperature $T_{w} \approx 10^{4} \mathrm{~K}$, and approximating $\dot{M}_{w} \approx 2 \pi r\left(1.4 m_{\mathrm{H}}\right) v_{w, z} N_{\mathrm{H}, w}$, we infer a shielding column

$$
\begin{aligned}
N_{\mathrm{H}, w} \approx & 1.5 \times 10^{22} \beta_{0.3}^{1 / 2} \eta_{10}^{-1 / 2} \delta_{0.1}^{1 / 2} \chi_{3}^{-1} \xi^{1 / 2} \\
& \times \Delta v_{5}^{1 / 2} x_{-5}\left(\mathrm{H}_{2} \mathrm{O}\right)^{-1 / 2} r_{0.1}^{1 / 2}\left(T_{w} / 20 T_{c}\right)^{-1 / 2} \mathrm{~cm}^{-2} .
\end{aligned}
$$

For $r \lesssim r_{\text {out }}$ this value should not exceed the maximum shielding column $N_{\max }$ for collisional pumping (see $\S 3$ ). By substituting the observed value of $r_{\text {out }}$ in NGC 4258 and using the fiducial values of all the other parameters in equation (31), we obtain $N_{\mathrm{H}, w} \approx 2.5 \times 10^{22} \mathrm{~cm}^{-2}$, which is approximately equal to the value of $N_{\max }$ shown in Figure 5 for the corresponding cloud density $\left(n_{c 9} \approx 2\right)$, assuming $x_{-5}\left(\mathrm{H}_{2} \mathrm{O}\right) \approx 1$ (see Fig. 6). This suggests that in this case the interclump medium (i.e., the dusty wind) may provide most of the requisite shielding of the masing gas from the incident high-energy radiation, whereas the clumped component (i.e., the uplifted clouds) mainly contributes the high-density gas that is needed for the collisional pumping of the masers. All in all, even though a comprehensive dynamical calculation of a clumpy wind is evidently required to fully establish the applicability of this model, the results of our simple analysis indicate that this is likely to be a viable explanation of the maser distribution in a source like NGC 4258.

\section{SUMMARY AND DISCUSSION}

Water megamaser emission in AGNs is typically detected in Seyfert 2-like galaxies that are viewed at a large angle to the symmetry axis. Recent VLBI-imaging observations of several of these sources reveal that the masers generally appear as discrete features that lie in a thin disk of radius $r \sim 0.1-1 \mathrm{pc}$ and radial extent $\Delta r \lesssim r$. Spectroscopic data indicate that the masers rotate with a nearly Keplerian velocity distribution, with high-velocity red- and blueshifted satellite features appearing on either side of the projected disk diameter (the midline). The masers are believed to be pumped collisionally.

By comparing the maser-emitting volume that produces the satellite features in a source like NGC 1068 with the maximum volume permitted by velocity-coherence considerations, and on the basis of the spectral and temporal behavior of the maser flux in a source like NGC 4258, we argue that, independent of the exact maser excitation mechanism, the emitting gas is likely clumped. The clumps could correspond to velocity irregularities in a turbulent medium, but in our specific models we assume that the maser features in fact represent distinct dense clouds. We show that even two overlapping filamentary clumps near the midline of a Keplerian disk that are separated from each other (along the line of sight) by less than the velocitycoherence length can account, through self-amplification, for the entire flux of a maser feature in sources like NGC 1068 and NGC 4258. The observed flux variability can then be attributed to relative motions that bring the clumps into or out of alignment with each other along the line of sight. The clump overlap probability is maximized for edge-on viewing of the disk, especially in the case of saturated emission (for which the minimum cloud separation that is required to account for the observed high-velocity maser fluxes can approach the coherence length); we point out that this could explain the strong observational bias in favor of detecting $\mathrm{H}_{2} \mathrm{O}$ maser emission in galaxies that are observed at a large angle to the disk rotation axis.

The interpretation of $\mathrm{H}_{2} \mathrm{O}$ maser features in terms of aligned dense clumps has previously been proposed for Galactic sources (Deguchi \& Watson 1989; EMH91). One example is provided by W49N, the most luminous Galactic water maser source, whose brightest features have fluxes of a few times $10^{4}$ Jy (e.g., Gwinn 1994). Interestingly, these fluxes are within about an order of magnitude of the peak fluxes of the high-velocity maser features in sources like NGC 4258 and NGC 1068 when the distance is scaled from $\sim 11 \mathrm{kpc}$ to $\sim 6$ and $\sim 15 \mathrm{Mpc}$, respectively. Furthermore, the bright outbursts in $\mathrm{W} 49 \mathrm{~N}$ have been commonly attributed to chance alignments of individual maser features (e.g., EMH91), and it has even been argued that they involve distinct clouds (Boboltz et al. 1998). All in all, NGC 4258 resembles maser outbursts in $\mathrm{W} 49 \mathrm{~N}$ both in terms of the flux level and variability. Another example is provided by the $\mathrm{H}_{2} \mathrm{O}$ maser source in Orion, where polarization measurements of a strong flare have been interpreted in terms of aligned clumps in a rotating disk that is viewed edge-on (e.g., Matveenko, Graham, \& Diamond 1988; Abraham \& Vilas Boas 1994). A similar disk interpretation has also been proposed for other water maser sources associated with young stellar objects (e.g., Fiebig et al. 1996; Torrelles et al. 1998). These examples suggest that the basic properties of extragalactic water megamaser sources may be quite similar to those of their bright Galactic counterparts even if the circumstances under which they arise are unique to the AGN environment.

In this paper we focus attention on a class of models that attributes the maser emission to heating by the central continuum source. In order for water molecules to survive the intense irradiation at the observed positions of the masers, shielding by a dusty medium of column density $N_{\text {shield }} \gtrsim$ $10^{22}-10^{23} \mathrm{~cm}^{-2}$ is required. Gas densities in the range $\sim 10^{8}-10^{10} \mathrm{~cm}^{-3}$, with a temperature $\gtrsim 250 \mathrm{~K}$ and a fractional water abundance $\sim 10^{-5}-10^{-4}$, are also needed for optimal masing. In our interpretation of the megamaser disks we identify the outer radius $r_{\text {out }}$ of the maser emission region with the shielding column $N_{\max }$ above which the temperature drops below $\sim 250 \mathrm{~K}$ and collisional pumping of water molecules ceases. We incorporate the above constraints into a minimalist scenario for AGN maser disks, in which the disk represents a low-mass ring that consists of a dominant cloud component and possibly also of a more tenuous intercloud medium. In the simplest representation of this model the radiation reaches the clouds through the plane of the ring; we find that such a flat-disk model is consistent with the observations in NGC 1068 and may also 
be applicable to NGC 4258 if the water abundance exceeds (e.g., because of the evaporation of icy grain mantles) the value determined by equilibrium photoionization-driven chemistry. The required gas densities and space densities of the clumps in this scenario are consistent with the values inferred for BELR-type clouds in AGNs.

We note the striking similarity between the ring properties implied by this interpretation of the tilted maser ring in NGC 1068 and those attributed to the circumnuclear disk (CND) in the Galactic center. Various suggestions have been considered for the origin of the CND and of its welldefined inner edge (e.g., Jackson et al. 1993), and the study of analogous structures in AGN megamaser disks might help resolve these issues. In the case of the CND there is evidence for ongoing accretion through the disk at a rate of $\sim 3 \times 10^{-2} M_{\odot} \mathrm{yr}^{-1}$, which is similar to the values inferred in some of the megamaser disks. Furthermore, in the Galactic case it has been argued on the basis of several dynamical considerations that the ring is a short-lived $\left(<10^{5}\right.$ yr) structure (e.g., Genzel et al. 1994). If a similar conclusion applies to the extragalactic megamaser rings then this could have important implications to our picture of how mass accumulates in the centers of AGNs. In particular, could it be that, on the scales of the maser emission regions, most of the mass flows to the center through shortlived, comparatively low-mass disks whose axes may be significantly tilted with respect to the local symmetry axis (as determined, for example, by the nuclear jet)? If most of the mass in such disks is in the clumped component, as inferred in the CND and consistent with the above interpretation of the megamaser disk in NGC 1068, how exactly is the angular momentum of the inflowing gas transported away? In a source like NGC 1068, does there also exist a massive circumnuclear disk that is roughly perpendicular to the inner radio jet axis, as has been inferred from various other observations (e.g., Antonucci 1993; Gallimore et al. 1996a, 1997; Tacconi et al. 1997)? If a distinct massive disk indeed exists in this archetypal object, does it interact dynamically with the maser ring? Could the simultaneous presence of edge-on disks and tilted rings in Seyfert 2-like galaxies be relevant to the puzzling finding of reprocessed $\mathrm{K} \alpha$ lines in Seyfert 2 and narrow emission line galaxies (Turner et al. 1998)? Should the ring picture be found to adequately describe the maser observations, then these and other related questions would need to be critically addressed.

We also consider the case in which the disk is too massive to permit the central continuum radiation to reach the masing clumps through its plane. The apparent warp seen in the maser distributions of both NGC 4258 and NGC 1068 provides observational support for the notion that the masing gas intercepts the incident radiation above the midplane. We present a scenario that, unlike the homogeneous disk model of Neufeld \& Maloney (1995), does not require the disk to be physically warped. Our proposed model is based on the hydromagnetic disk-driven wind scenario we had previously applied to the interpretation of the molecular "tori" in Seyfert 2-like galaxies and to other phenomena in AGNs (e.g., KK94; Kartje \& Königl 1997). In this picture, the wind uplifts (by its ram pressure) and confines (by its magnetic pressure) dense, diamagnetic clouds that form near the disk surface. The maser emission originates in the dense clouds once they become exposed to the central continuum radiation, with the dusty regions of the wind providing much of the requisite shielding. The apparent warp may then represent an observational selection effect induced by the strong vertical density stratification that characterizes centrifugally driven winds. We analyze the ingredients of a self-contained "generic" model of a clumpy wind that transports the bulk of the angular momentum of the underlying accretion disk. Using representative parameters, we show that this model can yield optimal masing conditions in a source like NGC 4258. We also demonstrate that the mass accretion rate implied by a hydromagnetic wind-driving disk model of this type is consistent with the ADAF interpretation of the spectrum of this source (Lasota et al. 1996).

The spiral shock model of Maoz \& McKee (1998) provides an alternative to the continuum-irradiation picture of megamaser disks. The shock scenario does not conflict with the discrete clumps interpretation presented in this paper since the maser emission in the clumps could, in principle, be excited by shocks. In fact, the relative motion of distinct clumps is still the most natural explanation of the strong flux variability exhibited by a source like NGC 4258. One attractive feature of the spiral shock model is that it readily accounts for the apparent tendency of the redshifted highvelocity maser features in the existing sample of megamaser sources to dominate over the blueshifted ones. There are, however, reported cases where the blueshifted features dominate (e.g., NGC 3079; Trotter et al. 1998), including a case (NGC 5793) where the flux of a redshifted feature decreased from being twice as large as the blueshifted maser flux to below the detection threshold on a timescale of $\sim 1$ month (Hagiwara et al. 1997). In these instances it is likely that the effects of cloud self-amplification, rather than the geometry of any shocks that might be present, control the appearance of the source. This conclusion is supported, in the case of NGC 3079, by the apparent fading and lighting up of certain maser features between consecutive observations, and it remains valid even if the maser emission in this source is excited by some other mechanical means (e.g., by the impact of a misaligned jet on the disk, as proposed by Trotter et al. 1998) rather than by spiral shocks. Further insights into the excitation mechanism of the $\mathrm{H}_{2} \mathrm{O}$ megamasers might be provided by studies of other masing molecules, such as $\mathrm{OH}$. In fact, it is now becoming clear that at least some of the $\mathrm{OH}$ maser emission detected in extragalactic sources is associated with the nuclei of active galaxies (e.g., Trotter et al. 1997; Lonsdale et al. 1998), and in some cases (e.g., NGC 1068; Gallimore et al. 1996b) it even appears to originate in the same material that produces the water maser radiation. These studies may be expected to lead to a comprehensive model of the megamaser emission in AGNs and could shed new light on the nature of the associated disks.

We thank G. Ferland for assistance with the use of CLOUDY, R. Antonucci, R. Barvainis, G. Ciolek, C. Gwinn, D. Hollenbach, J. Moran, T. Oka, and W. Watson for valuable conversations and correspondence, and the anonymous referee for insightful comments. A. K. also thanks T. Mazeh and $\mathrm{H}$. Netzer for helpful discussions and hospitality at Tel Aviv University during part of this work. This research was supported in part by NASA grants NAG5-2766 and NAG5-3687 (University of Chicago) and NAG5-3010 and NAG5-7031 (University of Kentucky). 


\section{APPENDIX A}

\section{RADIATION-INDUCED WARPING INSTABILITY IN A HYDROMAGNETIC WIND-DRIVING ACCRETION DISK}

In $\S 5$ we discuss a clumpy, accretion disk-driven hydromagnetic wind model for water megamaser sources. We show that, under the assumption that a significant fraction of the disk angular momentum is carried away by the wind, the implied mass accretion rate is consistent with that inferred from the ADAF interpretation of the spectrum in NGC 4258 (Lasota et al. 1996). In contrast, the irradiated viscous disk scenario considered by Neufeld \& Maloney (1995) implies an accretion rate that is significantly smaller than the ADAF value. In this Appendix we point out that a similar wind-driving accretion disk model can also resolve the discrepancy between the ADAF interpretation and the radiation-induced warping instability scenario for the origin of the apparent warp in this source. ${ }^{9}$ One should, however, bear in mind that the warping instability model for homogeneous disks is clearly distinct from the uplifted-cloud interpretation of the apparent warp outlined in $\S 5$.

In the radiative instability mechanism, first studied by Petterson (1977) and more recently elaborated on by Pringle (1996) and Maloney et al. (1996a), a disk illuminated by a central radiation source becomes unstable to warping if it is optically thick to both absorption and emission. The origin of the warp is the torque exerted locally on the disk by the reemitted radiation, which is directed normal to the disk surface (in contrast to the absorbed flux, which, being radial, produces no torque). ${ }^{10}$ The net torque on any given annulus of the disk is nonzero if the annulus is warped, and this would generally occur if there is a radial gradient in either the tilt angle or the angle of the line of nodes of the disk. Pringle (1996), using a local stability analysis, showed that the instability in a steadily accreting source is expected to arise at radii greater than

$$
r_{\text {warp } \min }=\zeta(\psi / \epsilon)^{2} R_{\mathrm{S}},
$$

where $\psi=v_{2} / v_{1}$ is the ratio of the effective $(r, z)$ and $(r, \phi)$ viscosities, $\epsilon=L_{\mathrm{bol}} / \dot{M}_{\mathrm{acc}} c^{2}$ is the radiative efficiency, $R_{\mathrm{S}}=2 G M / c^{2}$ is the Schwarzschild radius, and the constant $\zeta$ is $\sim 8 \pi^{2}$. Maloney et al. (1996a) applied the radiation-induced warping instability to the interpretation of the apparent warp in NGC 4258 (e.g., Herrnstein et al. 1996a). They noted, however, that this interpretation is inconsistent with the advection-dominated accretion model for this source. The origin of the difficulty can be seen by estimating the radiative efficiency in equation (A1) on the assumption of advection-dominated accretion. Taking $L_{\mathrm{bol}} \approx 10^{42} \mathrm{ergs} \mathrm{s}^{-1}$ and adopting a viscosity parameter $\gtrsim 0.1$, one gets $\epsilon \lesssim 2 \times 10^{-2}$. Using this estimate and the fiducial value of $\zeta$ yields $r_{\text {warp, } \min } \gtrsim 2 \times 10^{5} \psi^{2} R_{\mathrm{S}}$, which exceeds $r_{\min } \approx 4 \times 10^{4} R_{\mathrm{S}}$ for $\psi \approx 1$. However, as was already emphasized by Pringle (1992), $v_{1}$ and $v_{2}$ are physically distinct quantities, so $\psi$ in general may well differ from 1 . This parameter may, in fact, be expected to be less than 1 in a disk where a centrifugally driven outflow is the dominant angular momentum transport mechanism. In particular, if one uses the " $\alpha$ prescription" to represent the two effective viscosities, then, while $\alpha_{2}$ (which likely corresponds to a real viscous stress) is $\lesssim 1, \alpha_{1} \approx\left(\left|v_{r}\right| / C\right)(r / h)$ [where $h$ is the disk scale height, $v_{r}$ is the radial inflow velocity, $C$ is the speed of sound, and $\alpha_{1}$ now describes the effective $(z, \phi)$ viscosity] could potentially be $\gg 1$ (since $\left|v_{r}\right| \lesssim C$ in such a disk and $r / h \gg 1$; see, e.g., Wardle \& Königl 1993). If the disk in NGC 4258 drives a hydromagnetic wind, it may thus, in principle, be both advection dominated and warped by a radiation-induced instability.

Another potentially relevant aspect of a disk-driven hydromagnetic wind is related to the results of the global stability analysis carried out by Maloney et al. (1996a), who generalized the local treatment of Pringle (1996). They showed that radiation-induced warping instabilities in AGNs would be dominated by modes that peak near the outer radius of the disk, identified as either the physical edge of the disk or the radius where the disk becomes optically thin to the bulk of the incident or reemitted flux. Since a hydromagnetic disk outflow is expected to be dusty beyond the sublimation radius (see KK94), the radial flux component at the disk surface (which enters into the calculation of the torque on the disk) would be attenuated by passage through the wind as a result of dust scattering and absorption (as well as of electron scattering). This would tend to shift the effective outer radius of the disk inward, with the exact location determined by the density profile and total mass outflow rate in the wind.

To summarize the discussion in this Appendix, a disk-driven hydromagnetic wind may reconcile the radiation-induced warping interpretation and the advection-dominated accretion model in a source like NGC 4258. It may also be expected to affect the location of a warp arising from this instability. A physical warp is a necessary ingredient of the irradiated homogeneous disk model of Neufeld \& Maloney (1995), but, as discussed in $\S \S 4$ and 5, one could, in principle, account for the megamaser emission from AGN disks even if they were not warped. It is also worth reiterating our conclusion in $\S 2.1$ that a radiatively heated maser disk in a source like NGC 4258 is likely clumped even if warping (rather than, say, wind ram pressure on clouds) is responsible for exposing the masing gas to the central continuum.

\footnotetext{
${ }^{9}$ It is worth noting that the apparent warps that have so far been observed in megamaser disks (as well as in the Galactic CND) are comparatively mild and do not directly support the proposition that the radiation-induced warping instability could account for the collimated "ionization cones" found in Seyfert 2 galaxies (see Pringle 1997).

${ }^{10}$ An analogous mechanism, involving the torque exerted by a wind evaporated from the irradiated disk surface, was proposed by Schandl \& Meyer (1994).
}

REFERENCES

Abraham, Z., \& Vilas Boas, J. W. S. 1994, A\&A, 290, 956

Antonucci, R. 1993, ARA\&A, 31, 473

Arav, N., Barlow, T. A., Laor, A., \& Blandford, R. D. 1997, MNRAS, 288, 1015

Arav, N., Barlow, T. A., Laor, A., Sargent, W. L. W., \& Blandford, R. D. 1998, MNRAS, 297, 990
Baldwin, J., Ferland, G., Korista, K., \& Verner, D. 1995, ApJ, 455, L119 Blandford, R. D., \& Payne, D. G. 1982, MNRAS, 199, 883

Boboltz, D. A., Simonetti, J. H., Dennison, B., Diamond, P. J., \& Uphoff, J. A. 1998, ApJ, 509, 256

Bottorff, M., Korista, K., Shlosman, I., \& Blandford, R. D. 1997, ApJ, 479, 200 
Braatz, J. A., Wilson, A. S., \& Henkel, C. 1996, ApJS, 106, 51 1997, ApJS, 110, 321

Brown, P. D., Charnley, S. B., \& Millar, T. J. 1988, MNRAS, 231, 409

Ceccarelli, C., Hollenbach, D. J., \& Tielens, A. G. G. M. 1996, ApJ, 471, 400

Chary, R., \& Becklin, E. E. 1997, ApJ, 485, L75

Collison, A. J., \& Watson, W. D. 1995, ApJ, 452, L103

Deguchi, S., \& Watson, W. D. 1989, ApJ, 340, L17

de Kool, M. \& Begelman, M. C. 1995, ApJ, 455, 448

Elitzur, M. 1992, Astronomical Masers (Dordrecht: Kluwer)

Elitzur, M., Hollenbach, D. J., \& McKee, C. F. 1989, ApJ, 346, 983 (EHM89)

1992, ApJ, 394, 221 (EHM92)

Elitzur, M., McKee, C. F., \& Hollenbach, D. J. 1991, ApJ, 367, 333 (EMH91)

Emmering, R. T., Blandford, R. D., \& Shlosman, I. 1992, ApJ, 385, 460

Ferland, G. J. 1996, Hazy, a Brief Introduction to Cloudy, University of Kentucky Department of Physics and Astronomy Internal Report

Fiebig, D., Duschl, W. J., Menten, K. M., \& Tscharnuter, W. M. 1996, A\&A, 310, 199

Gallimore, J. F., Baum, S. A., \& O’Dea, C. P. 1996a, ApJ, 464, 198

. 1997, Nature, 388, 852

Gallimore, J. F., Baum, S. A., O’Dea, C. P., Brinks, E., \& Pedlar, A. 1996b, ApJ, 462, 740

Genzel, R., Eckart, A., Ott, T., \& Eisenhauer, F. 1997, MNRAS, 291, 219

Genzel, R., Hollenbach, D., \& Townes, C. H. 1994, Rep. Prog. Phys., 57 417

Greenhill, L. J., Ellingsen, S. P., Norris, R. P., Gough, R. G., Sinclair, M. W., Moran, J. M., \& Mushotzky, R. 1997a, ApJ, 474, L103

Greenhill, L. J., \& Gwinn, C. R. 1997, Ap\&SS, 248, 261

Greenhill, L. J., Gwinn, C. R., Antonucci, R., \& Barvainis, R. 1996, ApJ, 472, L21

Greenhill, L. J., Henkel, C., Becker, R., Wilson, T. L., \& Wouterloot, J. G. A. 1995a, A\&A, 304, 21

Greenhill, L. J., Jiang, D. R., Moran, J. M., Reid, M. J., Lo, K. Y., \& Claussen, M. J. 1995b, ApJ, 440, 619

Greenhill, L. J., Moran, J. M., \& Herrnstein, J. R. 1997b, ApJ, 481, L23

Greenhill, L. J., Trotter, A. S., Bragg, A., Moran, J. M., \& Herrnstein, J. R. 1997c, BAAS, 191, 7813

Güsten, R., Genzel, R., Wright, M. C. H., Jaffe, D. T., Stutzki, J., \& Harris, A. I. 1987, ApJ, 318, 124

Gwinn, C. R. 1994, ApJ, 429, 253

Hagiwara, Y., Kohno, K., Kawabe, R., \& Nakai, N. 1997, PASJ, 49, 171

Haschick, A. D., Baan, W. A., \& Peng, E. W. 1994, ApJ, 437, L35

Hawarden, T. G., Israel, F. P., Geballe, T. R., \& Wade, R. 1995, MNRAS, 276,1197

Helfer, T. T., \& Blitz, L. 1995, ApJ, 450, 90

Herrnstein, J. R., Greenhill, L. J., \& Moran, J. M. 1996a, ApJ, 468, L17

Herrnstein, J. R., Greenhill, L. J., Moran, J. M., Diamond, P. J., Inoue, M. Nakai, N., \& Miyoshi, M. 1998a, ApJ, 497, L69

Herrnstein, J. R., Moran, J. M., Greenhill, L. J., Blackman, E. G., \& Diamond, P. J. 1998b, ApJ, 508, 243

Herrnstein, J. R., Moran, J. M., Greenhill, L. J., Diamond, P. J., Miyoshi, M., Nakai, N., \& Inoue, M. 1996b, in ASP Conf. Ser. 103, The Physics of LINERs in View of Recent Observations, ed. M. Eracleous, A. Koratkar, C. Leitherer, \& L. Ho (San Francisco: ASP), 193 . 1997a, ApJ, 475, L17

Herrnstein, J. R., Moran, J. M., Greenhill, L. J., Inoue, M., Nakai, N., Miyoshi, M., \& Diamond, P. 1997b, BAAS, 191, 2507

Iwasawa, K., et al. 1993, ApJ, 409, 155

Jackson, J. M., et al. 1993, ApJ, 402, 173

Kartje, J. F., \& Königl, A. 1997, in ASP Conf. Ser. 128, Mass Ejection from AGNs, ed. N. Arav, I. Shlosman, \& R. Weymann (San Francisco: ASP), 282
Kartje, J. F., \& Königl, A. 1999, in preparation

Kartje, J. F., Königl, A., Hwang, C.-Y., \& Bowyer, S. 1997, ApJ, 474, 630

Königl, A., \& Kartje, J. F. 1994, ApJ, 434, 446 (KK94)

Königl, A., Kartje, J. F., Bowyer, S., Kahn, S. M., \& Hwang, C.-Y. 1995, ApJ, 446, 598

Laor, A., \& Draine, B. T. 1993, ApJ, 402, 441

Lasota, J.-P., Abramowicz, M. A., Chen, X., Krolik, J., Narayan, R., \& Yi, I. 1996, ApJ, 462, 142

Levine, D. A., Figer, D. F., Morris, M., \& McLean, I. S. 1995, ApJ, 447, L101

Lonsdale, C. J., Lonsdale, C. J., Diamond, P. J., \& Smith, H. E. 1998, ApJ, 493, L13

Maloney, P. R., Begelman, M. C., \& Pringle, J. E. 1996a, ApJ, 472, 582

Maloney, P. R., Hollenbach, D. J., \& Tielens, A. G. G. M. 1996b, ApJ, 466, 561

Maoz, E. 1995, ApJ, 447, L91

1998, ApJ, 494, L181

Maoz, E., \& McKee, C. F. 1998, ApJ, 494, 218 (MM98)

Matveenko, L. I., Graham, D. A., \& Diamond, P. J. 1988, Soviet Astron. Lett., 14,468

Miyoshi, M., Moran, J., Herrnstein, J., Greenhill, L., Nakai, N., Diamond, P., \& Inoue, M. 1995, Nature, 373, 127

Moran, J. M., Greenhill, L. J., Herrnstein, J. R., Diamond, P. J., Miyoshi, M., Nakai, N., \& Inoue, M. 1995, Proc. Natl. Acad. Sci. USA, 92, 11427

Nakai, N., Inoue, M., Miyazama, K., Miyoshi, M., \& Hall, P. 1995, PASJ, 47,771

Neufeld, D. A., \& Maloney, P. R. 1995, ApJ, 447, L17

Neufeld, D. A., Maloney, P. R., \& Conger, S. 1994, ApJ, 436, L127

Papaloizou, J. C. B., Terquem, C., \& Lin, D. N. C. 1998, ApJ, 497, 212

Petterson, J. A. 1977, ApJ, 216, 827

Pier, E. A., Antonucci, R. R. J., Hurt, T., Kriss, G., \& Krolik, J. H. 1994, ApJ, 428, 124

Pier, E. A., \& Voit, G. M. 1995, ApJ, 450, 628

Pringle, J. E. 1992, MNRAS, 258, 811 1996, MNRAS, 281, 357 1997, MNRAS, 292, 136

Rees, M. J. 1998, in Black Holes and Relativity, ed. R. Wald (Chicago: Univ. Chicago Press), 79

Schandi, S., \& Meyer, F. 1994, A\&A, 289, 149

Shakura, N. I., \& Sunyaev, R. A. 1973, A\&A, 24, 337

Tacconi, L. J., Gallimore, J. F., Genzel, Schinnerer, E., \& Downes, D. 1997, Ap\&SS, 248, 59

Torrelles, J. M., Gómez, J. F., Rodríguez, L. F., Curiel, S., Anglada, G., \& Ho, P. T. P. 1998 , ApJ, 505, 756

Trotter, A. S., Greenhill, L. J., Moran, J. M., Reid, M. J., Irwin, J. A., \& Lo, K.-Y. 1998, ApJ, 495, 740

Trotter, A. S., Moran, J. M., Greenhil, L. J., Zheng, X.-W., \& Gwinn, C. R. 1997, ApJ, 485, L97

Turner, T. J., George, I. M., Nandra, K., \& Mushotzky, R. F. 1998, ApJ, 493, 91

Wallin, B. K., \& Watson, W. D. 1997, ApJ, 476, 685

Wallin, B. K., Watson, W. D., \& Wyld, H. W. 1998, ApJ, 495, 774 1999 , ApJ, in press

Wardle, M., \& Königl, A. 1993, ApJ, 410, 218

Watson, W. D., \& Wallin, B. K. 1994, ApJ, 432, L35

Wilkes, B. J., Schmidt, G. D., Smith, P. S., Mathur, S., \& McLeod, K. K. 1995, ApJ, 455, L13

Wilson, A. S., Braatz, J. A., \& Henkel, C. 1995, ApJ, 455, L127 